\section{Contact-Initiated Shared Control Strategies for Four-Arm Supernumerary Manipulation with Foot Interfaces}

The International Journal of Robotics Research

$\mathrm{XX}(\mathrm{X}): 1-33$

(CThe Author(s) 2020

Reprints and permission:

sagepub.co.uk/journalsPermissions.nav

DOI: 10.1177/ToBeAssigned

www.sagepub.com/

SAGE

\author{
Walid Amanhoud ${ }^{\star 1}$, Jacob Hernandez Sanchez ${ }^{\star 1,2}$, Mohamed Bouri ${ }^{\dagger, 3}$, and Aude Billard ${ }^{\dagger 1}$
}

\begin{abstract}
In industrial or surgical settings, to achieve many tasks successfully, at least two people are needed. To this end, robotic assistance could be used to enable a single person to perform such tasks alone, with the help of robots through direct, shared or autonomous control. We are interested in four-arm manipulation scenarios, where both feet are used to control two robotic arms via bi-pedal haptic interfaces. The robotic arms complement the tasks of the biological arms, for instance, in supporting and moving an object while working on it (using both hands). To reduce fatigue, cognitive workload, and to ease the execution of the foot manipulation, we propose two types of assistance that can be enabled upon contact with the object (i.e., based on the interaction forces): autonomous-contact force generation and auto-coordination of the robotic arms. The latter relates to controlling both arms with a single foot, once the object is grasped. We designed four (shared) control strategies that are derived from the combinations (absence/presence) of both assistance modalities, and we compared them through a user study (with 12 participants) on a four-arm manipulation task. The results show that force assistance positively improves human-robot fluency in the four-arm task, the ease of use and usefulness; it also reduces the fatigue. To make this dual-assistance approach the preferred and more successful among the proposed control strategies, this force is also a crucial factor when controlling both robots with one foot.
\end{abstract}

\title{
Keywords
}

Telerobotics, Haptics and Haptic Interfaces, Physical Human-Robot Interaction, Human Performance Augmentation

\section{Introduction}

Many demanding tasks could be performed by a single human if the human could control more than two arms. This is the case in industry where a person works on an object that has to be held in a particular position and orientation (see Figure 1), or in surgery where the surgeon needs assistants to manipulate cameras or other surgical tools (Fuchs (2002)). By controlling multiple arms (via Supernumerary Robotic Limbs - SRL), the operator could perform these tasks alone, avoiding mistakes and misunderstandings that can occur when working in team. Furthermore, this would enable human resources to be reallocated and freed for other tasks. The use of robotic assistance is a promising solution in this context. A robot can assist a worker in performing a task through direct, autonomous, or shared control. With direct control, the user fully controls the robot, either by manual contact or via tele-operation. Tele-operation usually involves master-slave systems (Niemeyer et al. (2016)). Master interfaces can range from simple joysticks to voice systems, foot interfaces, or sensors that measure muscular or brain activities. Tele-operation is used in many applications to enable interaction with scaled, hazardous, or inaccessible environments such as for space exploration (Sheridan (1993)), minimally invasive surgery (Guthart and Salisbury (2000)), or search and rescue (Hirche et al.

\footnotetext{
${ }^{*}$ Contributed equally to this work

${ }^{\dagger}$ Equal senior authorship

${ }^{1}$ Learning Algorithms and Systems Laboratory (LASA), Swiss Federal School of Technology in Lausanne EPFL, Lausanne, Switzerland

${ }^{2}$ Biorobotics Laboratory (BIOROB), Swiss Federal School of Technology in Lausanne EPFL, Lausanne, Switzerland

${ }^{3}$ Translational Neural Engineering Laboratory (TNE), Swiss Federal Institute of Technology (EPFL), Geneva, Switzerland.
}

\section{Corresponding author:}

Walid Amanhoud, LASA Laboratory, Swiss Federal School of Technology in Lausanne EPFL, Switzerland

Jacob Hernandez Sanchez, BIOROB Laboratory, Swiss Federal School of Technology in Lausanne EPFL, Switzerland

Email: walid.amanhoud@epfl.ch

jacob.hernandezsanchez@epfl.ch 

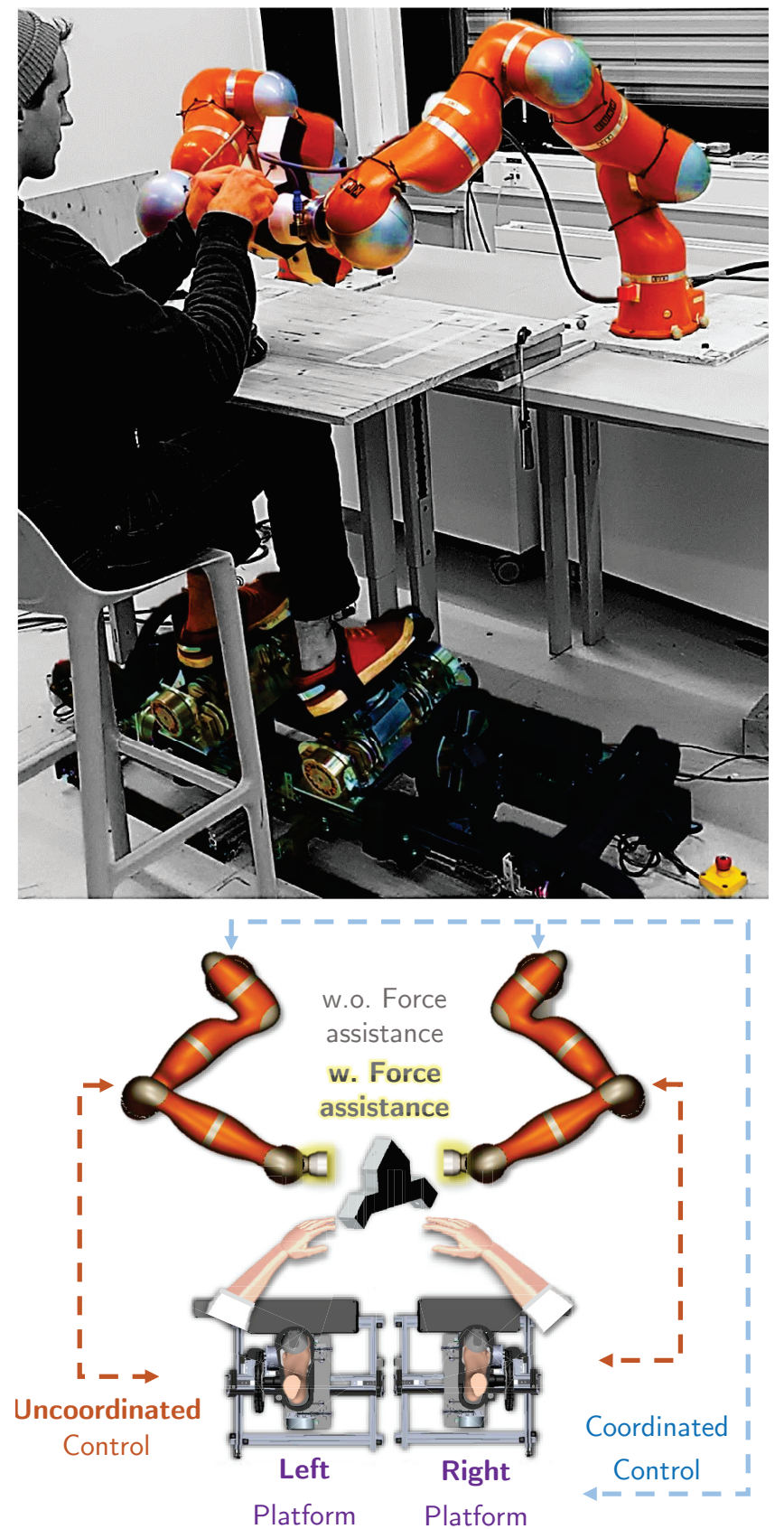

Figure 1. Four-arm shared manipulation of an object. A human operator works on an object with his two hands. Two robotic arms assist the operator in supporting and moving the object. These two robotic arms are controlled with one or two feet (depending on the shared control strategy) by using haptic robotic interfaces with five active degrees of freedom (DoF). We designed four control strategies. In this example, the user controls the left robot with the left foot and the right robot with the right foot. Once the grasp is complete, the robots generate autonomous forces to reinforce the grasp of the person (see control strategy $U+F$ in section 2.5).

(2003)). With conventional tele-operations, the robot has no autonomy or intelligence. Whereas, in tasks where the operator has to be physically involved in the execution (e.g., using both hands) while simultaneously controlling slave devices through master interfaces (see Figure 1), the cognitive workload can become substantial. In this case, providing robots with full autonomy is an ideal solution, as the robots can execute a task autonomously, without the need for human intervention (Brett et al. (2007); Parietti et al. (2015)). However, autonomous robots are still limited in their flexibility and dexterity when operating in uncertain and complex environments, i.e., humans should still be involved. Minimum human intervention can be achieved via supervisory control (Niemeyer et al. (2016)), where the human is involved in only the decision-making process, providing high-level commands to the robot that completes the (sub-)task autonomously (Shirkhodaie (2002)). With shared control, however, the worker and the robot are both involved in the execution of the task. Shared control is often presented as an intermediate level between direct and supervisory control. Shared control tele-operation appears in various forms in the literature, but according to Abbink and Mulder (2010), two main categories can be distinguished: input-mixing shared control and haptic shared-control. In the input-mixing category, the input from the human and the autonomous system are mixed at the level of the slave controller. They are mixed usually either in an overlapping or complementary way. The overlapping way is represented mainly by blending strategies where a weighted combination of the input defines the arbitration between the human and the autonomous system and enables an adaptive assistance level to be achieved (Hansson and Servin (2010); Goil et al. (2013); Storms and Tilbury (2014); Dragan and Srinivasa (2013); Gopinath et al. (2016); Muelling et al. (2017)). Conversely, in the complementary way, the human and the robot control different sub-spaces of the task, without affecting each other. This can be achieved by acting on different sub-spaces of a task, such as on different degrees of freedom (Abi-Farraj et al. (2016)) or using null-space control-strategies that exploit robot redundancies (Wang et al. (2015)). Haptic shared-control follows a different approach, where the human and the robot share the control of a task and the human is assisted by applying forces at the master device. Through a haptic interaction, the operator can be fully aware of the robot's intentions and express their own control intentions by choosing to conform or not with the robot. Furthermore, haptic feedback is particularly relevant for supernumerary manipulation. The perception of interaction force in conjunction with the perception of the robot's displacement during task execution contributes to engaging the user and to their feeling of embodiment (Segura Meraz et al. (2018)). Rosenberg (1993) first introduced the concept of virtual fixtures. Virtual fixtures can be seen as virtual elements, such as walls, velocity fields, or tubes that provide passive/active guidance to the human during the task execution (e.g., to 
follow a desired trajectory). The work of Rosenberg (1993) aroused interest in the field of tele-operation leading to further research in the use of virtual guidance (Selvaggio et al. (2016); Abi-Farraj et al. (2016, 2018)). In particular, Boessenkool et al. (2012) highlighted the benefits of haptic shared-control in terms of task performance (e.g., time completion, accuracy, contact force), control effort and cognitive workload, especially in free-motion phases. However, these benefits usually rely on an accurate task model and sensors' information. Inaccuracies between the task model and the real environment can lower the quality of the performance of the shared-control system, especially in constrained contact tasks (Smisek et al. (2015), van Oosterhout et al. (2015)). Therefore, in the aforementioned works, various shared-control strategies are used. They show that developing a proper strategy seems to be mainly task-dependent (Passenberg et al. (2010)). However, it is also essential to include the human aspect in the development process. In particular, criteria such as intuitiveness, fatigue, cognitive load, or comfort have to be considered. This is particularly true in cases where the worker is directly involved in the execution of the task (e.g., through physical interaction) while controlling the slave arms, and/or the control a complex system with multiple degrees of freedom such as a multi-arm system. Shared-control tele-operation of a multi-arm system has been considered in a few works in the literature, especially recently such as in Ferraguti et al. (2015a); Selvaggio et al. (2018), where input mixing and/or haptic shared-control strategies are used to control a dual-arm system. A multi-arm system can also benefit from shared control to enable coordination behaviours between the arms, which is particularly suitable for symmetric tasks such as supporting and moving a load. This is the case in Laghi et al. (2018) where a dual-arm system, tele-operated with hand gestures, switches to a coordinated behaviour controlled with one hand, once a particular gesture is detected. Similarly, in Rakita et al. (2019), assistance is provided to perform various coordinated tasks with the arms of a humanoid robot tele-operated with the arms of the user. The assistance is activated when the intention to perform a certain type of coordinated task is recognized from the motion of both hands (based on a predefined bi-manual action vocabulary). The coordination strategies proposed in Laghi et al. (2018) and Rakita et al. (2019) are enabled based on the human input and, in particular, on hand gestures or recognizable bi-manual patterns during distant (tele-operated) actions. In human-robot collaborative scenarios, however, strategies should also account for the physical interaction with the robotics systems, as one or more persons are involved in the execution of the task. For this reason, in scenarios combining both human-robot collaboration (physical interaction in close proximity) with tele-operation (e.g., via foot interfaces), the hands are busy with part of the task. Hence, the coordination strategies for the robotic manipulators need to be adapted to convey the human's intention to the robots through other input signals. For instance, the sensed interaction forces with the environment (i.e., the contact information), which are not considered in the related aforementioned works, constitute a suitable alternative in order to understand when assistance should be provided and to design proactive shared-control strategies. Additionally, by incorporating the contact information to trigger the robot assistance needed, special conditions of the operator, such as limited situational awareness or sensory-motor disorders, could be addressed.

In this work, we are interested in a four-arm manipulation scenario where two supernumerary robotic arms assist the operator in carrying and moving an object on which they work with both hands, see Figure 1. To this end, the feet of the operator are used to control each robotic arm through grounded kinesthetic haptic foot-interfaces. To enable and ease the four-arm solo manipulation of the object and to reduce the cognitive workload, we developed (shared) control strategies that provide assistance upon contact with the object, considering contact forces. This work is the continuation of our previous work in Hernandez Sanchez et al. (2019), where we introduced the concept of a world-grounded kinesthetic haptic foot-interface for four-arm manipulation. In Hernandez Sanchez et al. (2019), we validated the foot interface as a haptic device to be used simultaneously with both feet (in-obstructive footprint), and we defined the requirements in terms of work space and nominal forces/torques. The kinematic information of the foot robotic platforms can be found in Figure 2. The robotic arms were purely tele-manipulated (i.e., no assistance) through the foot interfaces by using dynamical system-based impedance control. Dynamical systems (DS) are used especially for their time-invariance property that provides rapid reactivity and on-the-fly re-planning of trajectories (Mirrazavi et al. (2016)). They have been used to control for impedance when making contact with an object (Salehian and Billard (2018); Kronander and Billard (2015)), or when physically interacting with people (Khoramshahi et al. (2018)), but also to control explicitly for the amount of force applied after contact with an object (Amanhoud et al. (2019)). In this paper, we exploit dynamical systems to design shared-control strategies that provide assistance once 
the contact with the object is made. Two types of assistance are proposed: autonomous-contact force generation to hold the object and auto-coordination of the robotic arms to ease the manipulation of the object. To evaluate the benefits of these two assistance modalities, four control-strategies are designed and compared with each other through a user study on a four-arm manipulation task. The four arms comprise the two biological arms and two robotic arms controlled through foot interfaces, see Figure 1. We consider a four-arm manipulation task, in which the two robotic arms are tasked to hold an object in the air while the user's natural arms are tasked to work on the object. The user can control the position of the object through the bi-pedal interface. Robotic assistance is necessary to prevent the object from falling, as the user applies forces on it during the manipulation, and to compensate for lack of coordination of the robot's motion when driven by the user's feet.

The contributions of this paper are two-fold:

- We designed four shared-control strategies for two foot-controlled supernumerary robotic arms that provide coordination and maintain a robust grasp. The four control-strategies combine two assistance modalities: coordination assistance and force assistance. The coordination is intended to mentally simplify the task (gestalt) as if the position of the object is directly being controlled by the pose of the foot. The force assistance is for picking up and holding the object, as well as balancing the forces bilaterally to maintain the grasp while moving the object. Delegating the robustness of the grasp (via force assistance) to the autonomy is intended to simplify, both mentally and physically, the operator's task so that they can focus only on moving the object with their feet and can prioritize the task of the hands.

- We conduct a systematic user evaluation of the four control-strategies that feature auto-coordination and autonomous force generation and their interactions. We quantify the importance of each form of assistance in terms of efficiency and success rate. We also assess how each form of assistance influences the fluency of the tasks and user's perceived task load. This evaluation is not specific to the experimental set-up and offers insight on the applicability of assistive control to supernumerary robotic limbs for tasks of holding, supporting, and positioning loads. Finally, we highlight the particular differences and consequences of using feet, as opposed to hands, in shared teleoperation for bi-pedal tasks.

We present the control strategies in Section 2. We explain the experimental-evaluation protocol in Section 3 and we provide the results in Section 4. We discuss the method, the results, and planned future works in Section 5 and finally conclude in 6 . The index for multimedia extensions is detailed in Appendix A. Furthermore, please note that we present all the technical details for implementation (i.e., values of parameters, thresholds, gains and matrices) in Appendix B.

\section{Method}

In this section, we present four control strategies for evaluating the effect of two types of assistance provided once an object is grasped: autonomous-contact force generation and auto-coordination of the robotic arms. We use superscript $i=\{L, R\}$ to represent the left or right robot/master foot interface (with respect to the human point of view, see Figure 1).

\subsection{Robots' Dynamics and Control}

The dynamics of a $N$ degrees of freedom robotic arm in the six-dimensional Cartesian space is given by

$$
M_{X, R}^{i}\left(q^{i}\right) \ddot{X}^{i}+C_{X, R}^{i}\left(q^{i}, \dot{q}^{i}\right) \dot{X}^{i}=W_{R, c}^{i}-W_{R, m}^{i}
$$

with $\boldsymbol{q}, \dot{\boldsymbol{q}}^{i} \in \mathbb{R}^{N}$ the joint state position and velocity, and $\dot{\boldsymbol{X}}, \ddot{\boldsymbol{X}}^{i} \in \mathbb{R}^{6}$ the robot's end-effector state velocity and acceleration, derived from the first and second-order differential kinematics:

$$
\left\{\begin{array}{c}
\dot{X}^{i}=\left(\begin{array}{c}
\dot{x}^{i} \\
\omega^{i}
\end{array}\right)=J_{R}^{i}\left(q^{i}\right) \dot{q}^{i} \\
\ddot{X}=J_{R}^{i}\left(q^{i}\right) \ddot{q}^{i}+\dot{J}_{R}^{i}\left(q^{i}\right) \dot{q}^{i}
\end{array}\right.
$$

$\boldsymbol{J}_{\boldsymbol{R}}^{\boldsymbol{i}}\left(\boldsymbol{q}^{\boldsymbol{i}}\right) \in \mathbb{R}^{6 \times N}$ is the geometric Jacobian that maps the joint-state velocity to the linear $\left(\dot{x}^{i} \in \mathbb{R}^{3}\right)$ and angular $\left(\omega^{i} \in \mathbb{R}^{3}\right)$ robot's end effector velocities. $M_{X, R}^{i}\left(q^{i}\right), C_{X, R}^{i}\left(q^{i}, \dot{q}^{i}\right) \in \mathbb{R}^{6 \times 6} \quad$ correspond to the inertia and Coriolis/centrifugal matrices projected in the operational space, whereas $\boldsymbol{W}_{\boldsymbol{R}, \boldsymbol{c}}^{\boldsymbol{i}}=\left[\boldsymbol{F}_{\boldsymbol{R}, \boldsymbol{c}}^{\boldsymbol{i}^{\boldsymbol{T}}}, \boldsymbol{T}_{\boldsymbol{R}, \boldsymbol{c}}^{\boldsymbol{i}^{\boldsymbol{T}}}\right]^{T}$ and $\boldsymbol{W}_{\boldsymbol{R}, \boldsymbol{m}}=\left[\boldsymbol{F}_{\boldsymbol{R}, \boldsymbol{m}}^{\boldsymbol{i}^{\boldsymbol{T}}}, \boldsymbol{T}_{\boldsymbol{R}, \boldsymbol{m}}^{\boldsymbol{i}^{\boldsymbol{T}}}\right]^{T}$ are , respectively, the taskspace control and measured external wrench; they can be decomposed into linear forces $\left(\boldsymbol{F}_{\boldsymbol{R}, \boldsymbol{c}}^{\boldsymbol{i}}, \boldsymbol{F}_{\boldsymbol{R}, \boldsymbol{m}}^{\boldsymbol{i}} \in \mathbb{R}^{3}\right)$ and torques $\left(\boldsymbol{T}_{\boldsymbol{R}, \boldsymbol{c}}^{i}, \boldsymbol{T}_{\boldsymbol{R}, \boldsymbol{m}}^{i} \in \mathbb{R}^{3}\right)$. Finally, (1) assumes that the 
gravity forces $g_{X, \boldsymbol{R}}^{i}\left(\boldsymbol{q}^{i}\right) \in \mathbb{R}^{6}$ are already compensated for."

In this work, the linear control force $\boldsymbol{F}_{R, c}^{i}$ is computed to track a desired velocity profile $\dot{x}_{d}^{i} \in \mathbb{R}^{3}$ expressed as a time-invariant dynamical system. Dynamical systems have emerged as a powerful and flexible means of representing robot motions. Indeed, they can encode complex tasks from human demonstrations and, while being able to counter disturbances in real-time, they can generate smooth trajectories. A time-invariant DS can be tracked through any velocity-based controller. To guarantee a stable interaction with the environment, we use the DS-based impedance controller (Kronander and Billard (2015)) that ensures passivity:

$$
F_{R, c}^{i}=D^{i}\left(x^{i}\right)\left(\dot{x}_{d}^{i}-\dot{x}^{i}\right)=d_{1}^{i} \dot{x}_{d}^{i}-D^{i}\left(x^{i}\right) \dot{x}^{i}
$$

where $\boldsymbol{x} \in \mathbb{R}^{3}$ is the end-effector position, and $\boldsymbol{D}^{i}\left(\boldsymbol{x}^{i}\right) \in$ $\mathbb{R}^{3 \times 3}$ is a state-varying damping matrix, constructed such that the first eigenvector is aligned with the desired dynamics $\dot{x}_{\boldsymbol{d}}^{i}$ with positive eigenvalue $d_{1}^{i} \in \mathbb{R}^{+}$. The two other eigenvectors are orthogonal to $\dot{x}_{d}^{i}$, thus allowing selective compliance in the directions orthogonal to the task. This can be desirable for safer physical human-torobot interaction (pHRI) with the user. Indeed, safety and physical compliance are desirable in supernumerary robotic systems where the human and the robotic limbs work in close proximity within a concurrent workspace. Whereas, to control the end-effector's orientation, we choose the axisangle representation to describe the error, which avoids singularities, and we compute $T_{R, c}^{i}$ through a PD control law:

$$
T_{R, c}^{i}=K_{o}^{i} \tilde{\zeta}^{i}+D_{o}^{i}\left(\omega_{d}^{i}-\omega^{i}\right)
$$

where $\quad \tilde{\zeta}^{i} \in \mathbb{R}^{3}$ is the axis-angle representation corresponding to the orientation error between the measured end-effector orientation axis $z_{o}^{i}$ (normal to the end-effector plane) and a desired one $z_{o, d}^{i} \cdot \omega_{d}^{i} \in \mathbb{R}^{3}$ represents the desired angular velocity, and $\boldsymbol{K}_{\boldsymbol{o}}^{\boldsymbol{i}} \in \mathbb{R}^{3 \times 3}$ and $\boldsymbol{D}_{\boldsymbol{o}}^{\boldsymbol{i}} \in \mathbb{R}^{3 \times 3}$ act as stiffness and damping gain matrices.

The control wrench $\boldsymbol{W}_{\boldsymbol{R}, \boldsymbol{c}}^{\boldsymbol{i}}$ is finally converted to joint torques $\tau_{R, c}^{i} \in \mathbb{R}^{N}$ by using the geometric Jacobian $J_{R}^{i}\left(q^{i}\right)$ such that $\tau_{R, c}^{i}=J_{R}^{i}\left(q^{i}\right)^{T} W_{R, c^{i}}^{i}$

Due to the nature of the tasks performed by the supernumerary robotic limbs in this work (holding, supporting and translating an object, with allowed rotation in the sagittal plane), in our method, the linear Cartesian control (DS-based impedance control) is different than the orientation control (PD control). Indeed, these tasks demand high physical compliance and robustness to disturbances during the linear displacements (translations) rather than demanding control of the orientation (as the orientation is fixed by design to a single axis). The combination of high physical compliance in the directions orthogonal to the task with a task-aligned high impedance is possible due to the use of the state-varying damping matrix that is computed on the fly on the DS-based impedance control. Furthermore, the relevance of the DS-based combination of motion + open-loop autonomous force generation (for the proposed shared control strategies involving force assistance) was evaluated in previous work (Amanhoud et al. (2019)).

\subsection{Master Foot Interfaces' Dynamics and Control}

Similarly to section 2.1, we define the dynamics of a master foot interface in its five-dimensional joint space:

$M_{M}^{i}\left(\xi^{i}\right) \ddot{\xi}^{i}+C_{M}^{i}\left(\xi^{i}, \dot{\xi}^{i}\right) \dot{\xi}^{i}+g_{M}^{i}\left(\xi^{i}\right)=\tau_{M, c}^{i}-\tau_{M, m}^{i}$

where $\xi^{i}=\left[x^{i}, y^{i}, \theta^{i}, \phi^{i}, \psi^{i}\right]^{T} \in \mathbb{R}^{5}$ denotes the platform's state with $x^{i}, y^{i}, \theta^{i}, \phi^{i}, \psi^{i}$ representing, respectively, the $\mathrm{x}$, $\mathrm{y}$, pitch, roll and yaw coordinates along their corresponding linear $\left(x^{i}, y^{i}\right)$ and rotation axes $\left(\theta^{i}, \phi^{i}, \psi^{i}\right)$, see Figure 2. $M_{M}^{i}\left(\xi^{i}\right) \in \mathbb{R}^{5 \times 5}$ is the mass matrix, $C_{M}^{i}\left(\xi^{i}, \dot{\xi}^{i}\right) \dot{\xi}^{i} \in$ $\mathbb{R}^{3}$ the centrifugal and coriolis forces, and $g_{M}^{i}\left(\xi^{i}\right) \in \mathbb{R}^{5}$ represents the gravity torques. Furthermore, $\tau_{M, c}^{i} \in \mathbb{R}^{5}$ and $\tau_{M, m}^{i} \in \mathbb{R}^{5}$ represent the control and measured external torques respectively. The control proposed for the foot platform is simply composed of a spring and damping terms and an open-loop rendering of desired torques reflected from the environment:

$$
\tau_{M, c}^{i}=\tau_{d}^{i}-K_{M}^{i} \xi^{i}-D_{M}^{i} \dot{\xi}^{i}
$$

where $\tau_{d}^{i} \in \mathbb{R}^{5}$ are the desired reflected torques, and $\boldsymbol{K}_{M}^{i} \in$ $\mathbb{R}^{5 \times 5}$ and $\boldsymbol{D}_{M}^{i} \in \mathbb{R}^{5 \times 5}$ are the stiffness and damping matrices pushing the foot towards $\boldsymbol{\xi}^{i}=\mathbf{0}$. According to the type of mapping chosen (see Figure 7), the virtual stiffness of the degrees of freedom with position:velocity mappings can be high, resembling a joystick like spring. Whereas, for the position:position mappings there is no virtual stiffness; instead there is damping to smooth down the control with the foot.

${ }^{*}$ Gravity compensation is provided by the manufacturer of the robots used in this paper. 

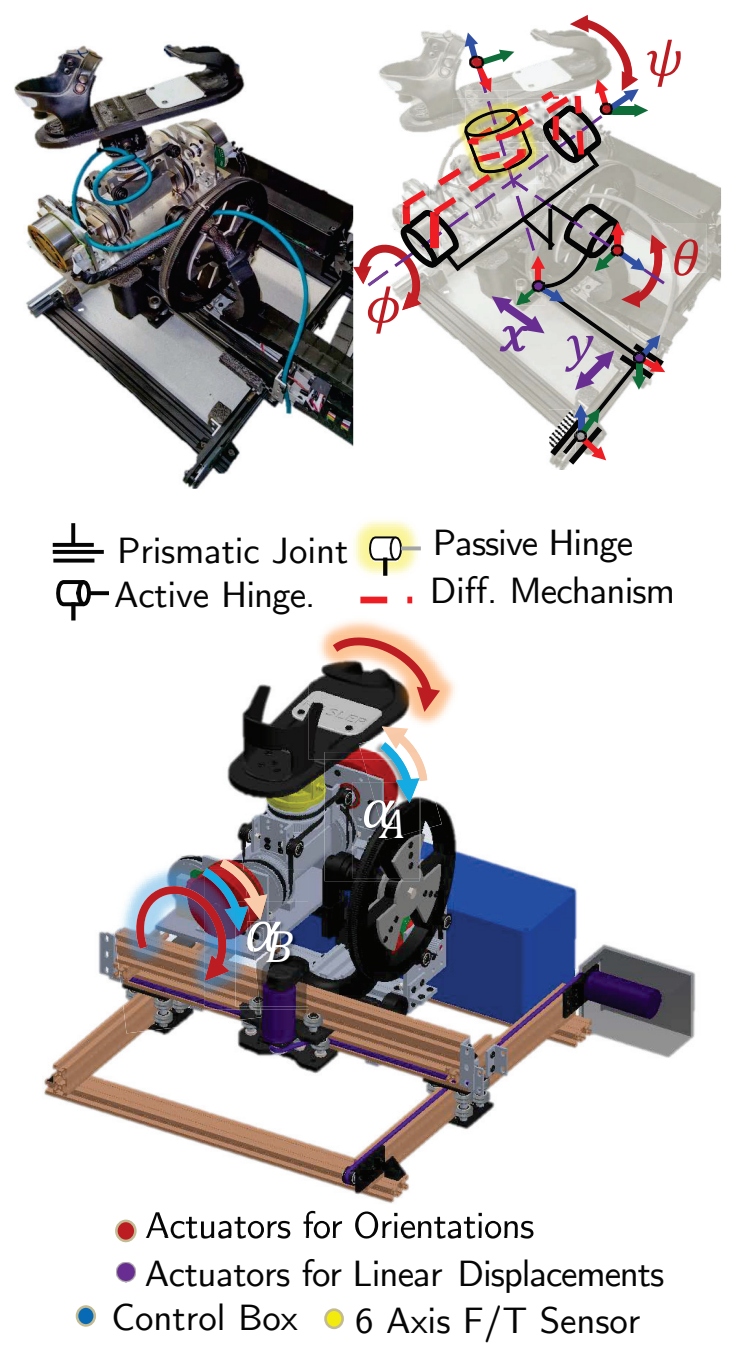

Figure 2. Kinematic model of the 5-DoF haptic foot platforms (c.f., Hernandez Sanchez et al. (2019)). The kinematic chain consists in a serial-parallel hybrid mechanism, where the first three joints joints $(y, x, \theta)$ are serial (prismatic, prismatic, revolute - gimbal) and the last two joints $(\phi, \psi)$, are achieved through a differential mechanism that maps the rotation of two coaxial motors into two orthogonal axes of rotation. Specifically, the roll rotation $(\phi)$ is enabled when the two motors (A and B) rotate in the same direction, whereas the yaw rotation $(\psi)$ is enabled when both motors rotate in opposite directions: $\phi=\alpha_{\mathrm{A}}+\alpha_{\mathrm{B}}$ and $\psi=\alpha_{\mathrm{A}}-\alpha_{\mathrm{B}}$, where $\alpha_{\mathrm{A}}$ and $\alpha_{\mathrm{B}}$ represent the angles of the front and rear motors, respectively. The belt-driven differential mechanism achieves two orthogonal axes of rotation due to idler pulleys that act as $90^{\circ}$ torque conveyors. Moreover, this mechanism enables us to duplicate the nominal torque of the transmission for isolated rotations.

\subsection{Control Strategies Overview}

The four (shared) control strategies result from the combinations (presence/absence) of the two assistance modalities considered in this work, namely, force and coordination assistance, and they are illustrated in Figure 3. The assistance modalities are activated once the contact of the object is made. Prior to contact, all control strategies behave the same, with each foot controlling separately its ipsilateral robotic arm (i.e., individual control phase, Figure $3 a)$. The operator is responsible for positioning and orienting the robotic arms to perform the grasp (Figure 3b). As soon as the interaction forces have surpassed a threshold (i.e., after contact) four control strategies can be distinguished:

\section{- Uncoordinated Control without Force Assistance (U,} Figure 3c1): This is the baseline where the operator uses their two feet to control the robotic arms. The operator must maintain the grasp, while moving and orienting the object to facilitate the task of the hands. The grasping forces are rendered in the feet.

- Uncoordinated Control with Force Assistance $(U+F$, Figure 3c2): The operator still controls the two robotic arms with their two feet but a force assistance is provided to keep a robust grasp. Consequently, the operator should focus only on orienting and positioning the object. The force rendered in the feet is now much less, as it is the error between the assisting contact forces and the measured endeffector forces.

- Coordinated Control without Force Assistance (C, Figure $3 c 3)$ : The control of both robots switch from two feet to a single foot (the dominant one) after the contact is done, and the two robots' movements are coordinated. The operator controls the position of the geometric center between both end effectors, as well as the opening (distance between both end effectors). The dual-arm grasp is controlled in the fifth degree of freedom of the platform (foot's self-rotation) and the net grasping force is also rendered in this axis. The purpose of coordination is to make a mental simplification so that the user feels as if they are controlling directly the pose of the object with their foot.

\section{- Coordinated Control without Force Assistance $(C+F$,} Figure 3c4): The operator also controls both robotic arms by using a single foot but, in this case, the force for keeping the grasp and holding the object while moving it is done autonomously. This is done in order to simplifiy the task, both mentally and physically.

In the uncoordinated control strategies (i.e., $U$ and $U+F$ ), to break the grasp, the operator has to move his feet away from each other along the left-right direction (Figure $3 \mathrm{~d}$ ). For strategy $U+F$ in particular, this means going against the haptic feedback (i.e., the rendered force error). In this case, once the measured contact forces are lower than a given threshold, force assistance is stopped and the autonomous grasp is released. Regarding the coordinated control strategies (i.e., $C$ or $C+F$ ), the grasp can be released with the fifth DOF of the foot platform (i.e, the one responsible for opening/closing, Figure $3 \mathrm{~d} 1$ ). The robotic arms remain 


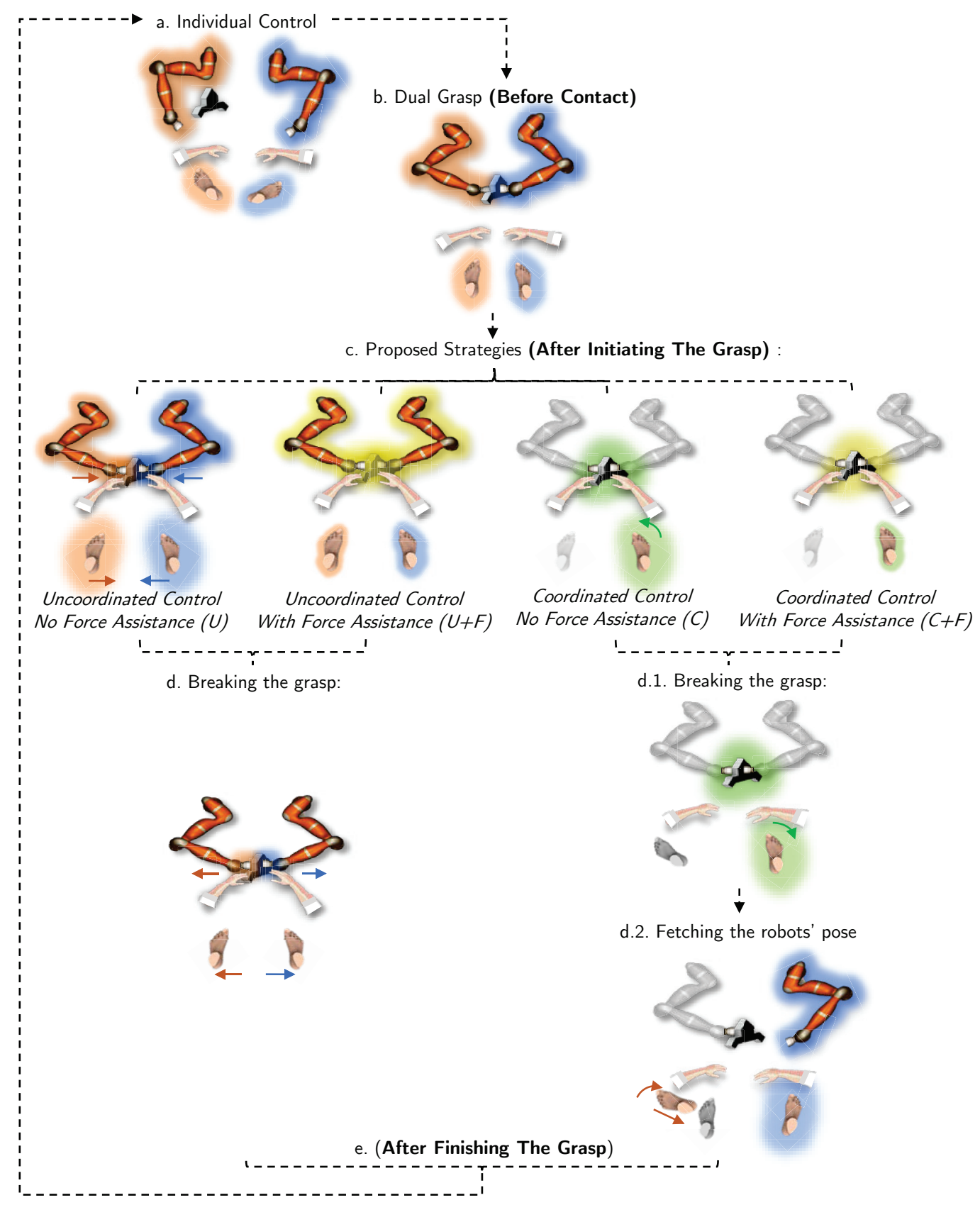

Figure 3. Overview of the proposed (shared) control strategies enabled once the grasp is made. Uncoordinated (or individual) control of the robotic arms by each foot is represented by orange and blue glows. After contact, the force assistance-modality is represented by a yellow glow (control strategies $U+F$ and $C+F$ ), and a green glow is associated with coordination assistance (control strategies $C$ and $C+F$ ).

coordinated until the distance between the two end-effectors exceeds a certain threshold. At that moment, to assure a stable transition, the feet have to fetch the robotic arms in the workspace of the foot platforms. This fetching phase is accompanied with haptic cues that push the feet to the required pose (Figure 3d2). When a distance threshold is met, then the individual control of the robotic arms is regained (Figure 3a).

The above-mentioned four control-strategies are described in detail in the following sections.

\subsection{Uncoordinated Robot Control without Force Assistance (U)}

In this control strategy, the two foot interfaces are used to control both robots. Each robot tracks a linear dynamical system whose attractor is obtained by mapping the associated foot pose to a desired attractor in the robots' world frame. To this end, we define $\dot{x}_{d}^{i}$ as follows:

$$
\dot{x}_{d}^{i}=A^{i}\left(x_{d}^{i}-x^{i}\right)
$$


where $\boldsymbol{A}^{i} \in \mathbb{R}^{3 \times 3}$ is a positive diagonal gain matrix with constant values, and $\boldsymbol{x}_{\boldsymbol{d}}^{\boldsymbol{i}} \in \mathbb{R}^{3}$ denotes the desired robot attractor designed such that

$$
\left(\begin{array}{c}
\boldsymbol{x}_{\boldsymbol{d}}^{\boldsymbol{i}} \\
\omega_{h}^{i}
\end{array}\right)=\boldsymbol{\Upsilon}_{\boldsymbol{p}}^{\boldsymbol{U}} \boldsymbol{\xi}^{\boldsymbol{i}}+\left(\begin{array}{l}
\boldsymbol{x}_{\boldsymbol{o}}^{\boldsymbol{i}} \\
0
\end{array}\right)
$$

$\boldsymbol{x}_{\boldsymbol{o}}^{\boldsymbol{i}} \in \mathbb{R}^{3}$ is a fixed attractor offset, and $\boldsymbol{\Upsilon}_{\boldsymbol{p}}^{\boldsymbol{U}} \in \mathbb{R}^{4 \times 5}$ is a tele-functioning matrix mapping the foot's pose (measured in the platform joint space) to the desired degrees of freedom in the robots' task space, when the robots are uncoordinated. As can be appreciated in Appendix B, this matrix considers transformations in the reference frames between the master device (foot platform) and the tele-manipulator, as well as with the scaling factors between the joints of foot robotic platform and the task coordinates of the end-effector of the robotic arm. Depending on the defined mappings for the foot gestures, $\Upsilon_{\boldsymbol{p}}^{U}$ can include position:position and/or position:velocity mappings between degrees of freedom. Here, $\omega_{h}^{i} \in\left[-\omega_{h, l i m}, \omega_{h, l i m}\right]$ with $\omega_{h, l i m}>0$ is an angular velocity input that enables self-rotation of the end-effector along $z_{o}^{i}$. A position:velocity mapping is used to control the self-rotation of the end-effector, as its range of motion is significantly larger than the range of motion of the human input (see Figure 7).

Concerning the orientation, $z_{\boldsymbol{o}, \boldsymbol{d}}^{\boldsymbol{i}}$ is fixed to a predefined direction for both robots, and $\boldsymbol{\omega}_{\boldsymbol{d}}^{\boldsymbol{i}}$ is defined as the sum of two terms, one term helps align $z_{o}^{i}$ with $z_{o, d}^{i}$ and a second term $\left(\omega_{h} z_{0}^{i}\right)$ enables self-rotation of the end-effector (see Appendix B for more details).

Furthermore, this control strategy enables the measured robot end-effector forces (obtained with force/torque sensors) to be reflected to the master interfaces, such that the human can perceive the robots' interaction with the environment. Therefore, we derive $\tau_{d}^{i}$ accordingly:

$$
\tau_{d}^{i}=\Upsilon_{F}^{U} F_{R, m}^{i}
$$

where $\boldsymbol{\Upsilon}_{\boldsymbol{F}}^{\boldsymbol{U}} \in \mathbb{R}^{5 \times 3}$ is the force tele-functioning matrix that maps the interaction forces of the environment to the foot through haptic feedback (rendered in the joint space of the foot platform). It also considers the differences in the reference frames of the pedal and the robot's end-effector. Depending on the choice of mapping for the foot gestures (see Figure 7), this matrix can include force:force or force:torque transformations between the joints of the foot platform and the end-effector of the robot. As can be seen in Appendix B, the interaction forces are conveyed to the user's feet with a scaling factor that changes depending on the control strategy.

The haptic feedback works selectively for the usercontrolled degrees of freedom that are relevant for keeping the grasp (i.e., linear translations). Indeed, this is to evoke a feeling of telepresence, while performing and keeping the grasp. Whereas, for the control of the object's self-rotation, a joystick-like virtual spring-damper is rendered in the axis of the platform that controls the orientation of the end-effector around the axis of the last joint of the manipulator with position:velocity mapping. Joystick-like means that a force of the spring-damper will push the user's foot to the resting position.

\subsection{Uncoordinated Robot Control with Force Assistance $(U+F)$}

In this control strategy, each robot is controlled through its corresponding foot interface, as in Section 2.4. However, force assistance is provided to support the object once the object is grasped. To this end, using our previous work in Amanhoud et al. (2019), (7) is modified as follows:

$$
\dot{x}_{d}^{i}=A^{i}\left(x_{d}^{i}-x^{i}\right)+\frac{F_{d}^{U, i}}{d_{1}^{i}} z_{o}^{i}
$$

where the desired contact force $F_{d}^{i}$ is set to

$$
F_{d}^{U, i}= \begin{cases}F_{T}^{U, i} & \text { grasped } \\ 0 & \text { not grasped }\end{cases}
$$

with $F_{T}^{U, i}>0$ the target force of robot $i$ when the object is grasped; i.e., persistent contact forces are detected from both robots' force-torque sensors. This grasp condition is given below:

$$
\begin{cases}\text { grasped } & \mu_{F}^{L} \geq \varepsilon_{F} \wedge \mu_{F}^{R} \geq \varepsilon_{F} \\ \text { not grasped } & \text { otherwise }\end{cases}
$$

where $\mu_{F}^{i}$ with $i=\{L, R\}$ is the mean value over a sliding window of size $n$ of the measured forces along the $z_{o}^{i}$ axis of the robot's end-effector, while $\varepsilon_{F} \geq 0$ is a force threshold.

The haptic feedback in (9) is modified to reflect the force error between the measured and desired contact forces at the master interface, such that

$$
\tau_{d}^{i}=\Upsilon_{F}^{U}\left(F_{R, m}^{i}+F_{d}^{U, i} z_{o}^{i}\right)
$$


This reduces the risks for the operator to unintentionally break the grasp, as the feet can compensate for this force error.

\subsection{Coordinated Robot Control with Force Assistance $(C+F)$}

In this control strategy, initially, when the object is not grasped, the two robots are controlled individually in an uncoordinated manner (i.e., as in Section 2.4). Once the object is grasped, both the force assistance and autocoordination of the robotic arms are activated to ease the support and movement of the manipulated object. During the coordination, a single foot interface is used at the same time to control both robots . Figure 4 highlights the switching mechanisms between uncoordinated and coordinated robot control with force assistance. In particular, to switch to the coordinated control, the grasp condition is used. To switch back to the uncoordinated control, a fetching mechanism is developed to increase safety and will be detailed later.

First, let us design $\dot{x}_{d}^{i}$ to enable auto-coordination of the arms and autonomous-contact force generation when the object is grasped. Using the work in Amanhoud et al. (2019), we introduce two auxiliary variables:

$$
x^{C}=\frac{x^{L}+x^{R}}{2} \quad x^{D}=x^{R}-x^{L}
$$

where $x^{C} \in \mathbb{R}^{3}$ and $x^{D} \in \mathbb{R}^{3}$, respectively, denote the robots' center position and distance vector. From (14), the following relations hold:

$$
\dot{\boldsymbol{x}}^{R}=\dot{\boldsymbol{x}}^{C}+\frac{\dot{\boldsymbol{x}}^{D}}{2} \quad \dot{\boldsymbol{x}}^{L}=\dot{\boldsymbol{x}}^{C}-\frac{\dot{\boldsymbol{x}}^{D}}{2}
$$

A coordination behaviour can be achieved between the robots by explicitly controlling a desired center position $x_{d}^{C}$ and distance vector $\boldsymbol{x}_{\boldsymbol{d}}^{D}$. To track these two entities, we use the desired linear dynamics below:

$$
\left\{\begin{array}{l}
\dot{x}_{d}^{C}=A_{C}\left(x_{d}^{C}-x^{C}\right) \\
\dot{x}_{d}^{D}=A_{D}\left(x_{d}^{D}-x^{D}\right)
\end{array}\right.
$$

where $\boldsymbol{A}_{\boldsymbol{C}} \in \mathbb{R}^{3 \times 3}$ and $\boldsymbol{A}_{\boldsymbol{D}} \in \mathbb{R}^{3 \times 3}$ are positive gain diagonal matrices. Assuming that the object is grasped, $\dot{x}_{d}^{C}$ and $\dot{x}_{d}^{D}$, respectively, enable us to control the object position and orientation.

Using (15) and our approach in Amanhoud et al. (2019) to generate contact forces to maintain the grasp, we can express

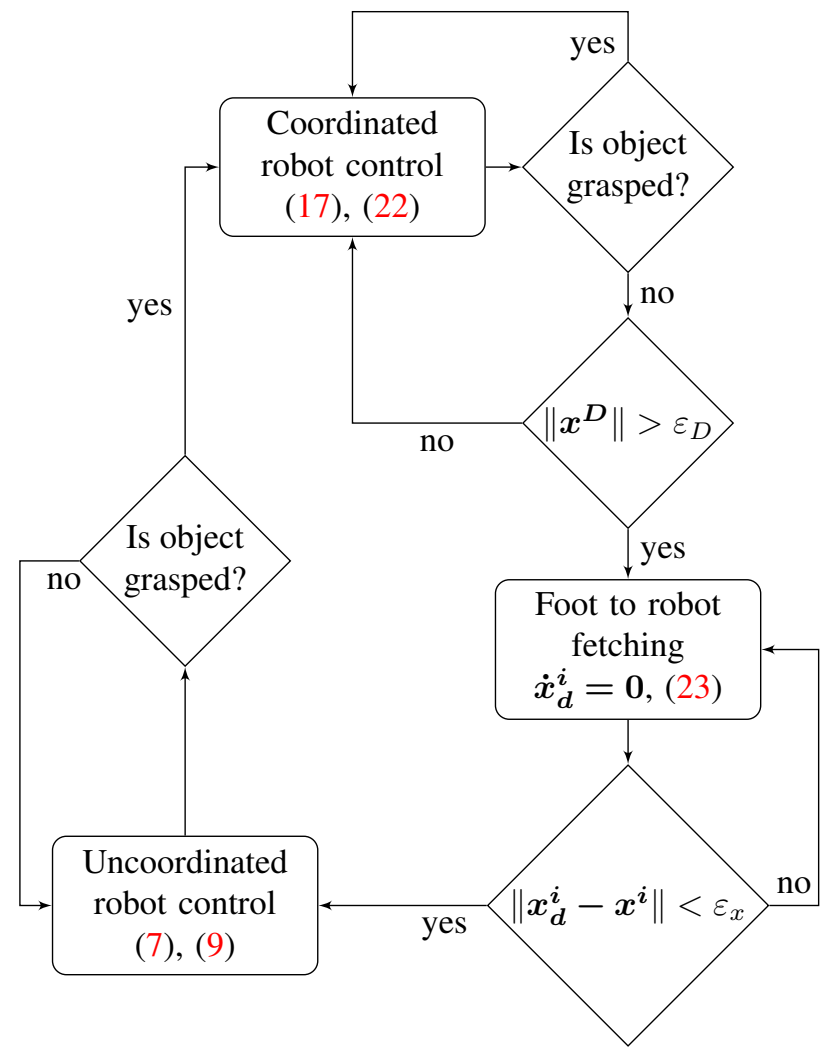

Figure 4. Flow-chart representation of $C+F$ control strategy with the switching mechanisms between coordinated and uncoordinated robot behaviours.

the desired robots' dynamics $\dot{x}_{d}^{i}$ as

$$
\dot{\boldsymbol{x}}_{\boldsymbol{d}}^{i}=\dot{\boldsymbol{x}}_{\boldsymbol{d}}^{C}+\tilde{\dot{x}}_{\boldsymbol{d}}^{D^{i}}+\frac{F_{d}^{C, i}}{d_{1}^{i}} \boldsymbol{n}^{i}+\dot{x}_{h} \boldsymbol{n}^{i}
$$

with

$$
\tilde{\dot{x}}_{d}^{D^{R}}=-\tilde{\dot{x}}_{d}^{D^{L}}=\frac{\dot{x}_{d}^{D}}{2} \quad n^{L}=-n^{R}=\frac{x_{d}^{D}}{\left\|x_{d}^{D}\right\|}
$$

The first two terms in (17) enable the robots to move in coordination. The third term generates contact force along the desired grasping direction $n^{i}$ obtained from $x_{d}^{D}$, such that

$$
\boldsymbol{x}_{\boldsymbol{d}}^{D}= \begin{cases}\boldsymbol{x}_{0}^{D} & \text { grasped } \\ \boldsymbol{P} \boldsymbol{x}^{D} & \text { not grasped }\end{cases}
$$

where $x_{0}^{D}$ is the initial distance vector measured after grasping the object, and $\boldsymbol{P} \in \mathbb{R}^{3 \times 3}$ is a projection matrix used to constrain the distance vector along a specific direction when the object is not grasped. The desired contact force $F_{d}^{C, i}$ is set to

$$
F_{d}^{C, i}= \begin{cases}F_{T}^{C, i} & \text { grasped } \\ 0 & \text { not grasped }\end{cases}
$$

where $F_{T}^{C, i}>0$ is the target force when the object is grasped. The last term in (17) is the human-input 
velocity along the desired grasping direction where $\dot{x}_{h} \in\left[-\dot{x}_{h, \text { lim }}, \dot{x}_{h, \text { lim }}\right]$ with $\dot{x}_{h, \text { lim }}>0$. It enables the human to break the grasp or squeeze more tightly the object.

In order to reduce human cognitive workload and fatigue, a single master foot interface is used to control the two robots; five degrees of freedom are available as human input. One is used to compute $\dot{x}_{h}$, and three are for defining the desired robots' center position $\boldsymbol{x}_{\boldsymbol{d}}^{\boldsymbol{C}}$. The remaining one is used to rotate the end-effectors, along a specific direction. These transformations are described in (21):

$$
\left(\begin{array}{c}
\boldsymbol{x}_{\boldsymbol{d}}^{\boldsymbol{C}} \\
\dot{x}_{h} \\
\omega_{h}
\end{array}\right)=\boldsymbol{\Upsilon}_{\boldsymbol{p}}^{\boldsymbol{C}} \boldsymbol{\xi}^{\boldsymbol{j}}+\left(\begin{array}{l}
\boldsymbol{x}_{\boldsymbol{o}}^{\boldsymbol{C}} \\
0 \\
0
\end{array}\right)
$$

where, similarly to $\Upsilon_{\boldsymbol{p}}^{\boldsymbol{U}}$ in (8), $\boldsymbol{\Upsilon}_{\boldsymbol{p}}^{\boldsymbol{C}} \in \mathbb{R}^{5 \times 5}$ is the pose telefunctionning matrix when the robots are coordinated, and $\boldsymbol{\xi}^{j}$ is the pose of the master interface $j$ used to control both robots. $x_{\boldsymbol{o}}^{C} \in \mathbb{R}^{3}$ is a fixed attractor offset. $\omega_{h} \in\left[-\omega_{h, l i m}, \omega_{h, l i m}\right]$ with $\omega_{h, l i m}>0$ is the angular velocity input used to rotate the object around the grasping direction $\boldsymbol{n}^{j}$, as in Section 2.4.

Regarding the orientation, the desired end-effector orientation axis $\boldsymbol{z}_{o, \boldsymbol{d}}^{\boldsymbol{i}}$ is set to $\boldsymbol{n}^{\boldsymbol{i}}$, and $\boldsymbol{\omega}_{\boldsymbol{d}}^{\boldsymbol{i}}$ also has two components, one to align $z_{o}^{i}$ with $z_{o, d}^{i}$ and another one $\left(\omega_{h} \boldsymbol{n}^{j}\right)$ to rotate the object along $\boldsymbol{n}^{j}$ (see Appendix B for more details).

Similarly, as in Section 2.5, by reflecting the force error between the measured and desired contact force to the master interface, the haptic feedback is also provided to avoid an unintentional break of the grasp:

$$
\boldsymbol{\tau}_{\boldsymbol{d}}^{i}= \begin{cases}\boldsymbol{\Upsilon}_{\boldsymbol{F}}^{C}\left(\boldsymbol{F}_{\boldsymbol{R}, \boldsymbol{m}}^{i}+F_{d}^{C, i} \boldsymbol{n}^{i}\right) & i=j \\ \mathbf{0} & \text { otherwise }\end{cases}
$$

where $\Upsilon_{\boldsymbol{F}}^{C} \in \mathbb{R}^{5 \times 3}$ is the force telefunctionning matrix acting as $\boldsymbol{\Upsilon}_{\boldsymbol{F}}^{U}$ in (9).

Finally, we previously discussed how the transition from uncoordinated to coordinated robot control occurs using the grasp condition in (12). To switch back to uncoordinated control, we proceed as illustrated in Figure 4. First, the human should break the grasp through $\dot{x}_{h}$. From there, the robots remain in coordinated control, as long as the distance between them (i.e., $\left\|\boldsymbol{x}^{D}\right\|$ ) does not exceed a threshold $\left(\varepsilon_{D}>0\right)$. If the threshold is exceeded, both robots stop moving $\left(\dot{\boldsymbol{x}}_{\boldsymbol{d}}^{i}=\mathbf{0}\right)$ until the human fetches them with the foot interfaces. The fetching phase consists in moving each foot until the distance between the desired attractor commanded through the foot (i.e., $\boldsymbol{x}_{\boldsymbol{d}}^{\boldsymbol{i}}$ in (8)) and the current robot position $x^{i}$ becomes lower than a threshold $\varepsilon_{x}>0$. To ease the fetching phase and to help the human 'find' the robots, haptic cues are provided using (23):

$$
\tau_{d}^{i}=\Omega_{F}^{C}\left(x^{i}-x_{d}^{i}\right)
$$

where $\Omega_{\boldsymbol{F}}^{\boldsymbol{C}} \in \mathbb{R}^{5 \times 3}$ is a telefunctionning matrix mapping the position error to the forces at the foot interface. This transition mechanism improves safety by avoiding overly rapid robot motions that would occur if the robots immediately switch to uncoordinated control, after breaking their grasp.

\subsection{Coordinated Robot Control without Force Assistance (C)}

This control strategy enables auto-coordination of the robotic arms upon contact with the object. This is the same as in Section 2.6, except (17) that is simplified to

$$
\dot{x}_{d}^{i}=\dot{x}_{d}^{C}+\tilde{\dot{x}}_{d}^{D^{i}}+\dot{x}_{h} n^{i}
$$

(22) is modified to reflect, as feedback to the master interface, the measured interaction forces instead of the force error:

$$
\boldsymbol{\tau}_{\boldsymbol{d}}^{i}= \begin{cases}\boldsymbol{\Upsilon}_{\boldsymbol{F}}^{C} \boldsymbol{F}_{\boldsymbol{R}, \boldsymbol{m}}^{\boldsymbol{i}} & i=j \\ \mathbf{0} & \text { otherwise }\end{cases}
$$

\section{Experimental Evaluation}

In this section, we evaluate the four control-strategies described above in a four-arm manipulation (4A) task. Our objective is to evaluate the benefits of the two types of assistance considered in this work: autonomous-contact force generation and auto-coordination of the robotic-arms. To this end, a user study was conducted. The details about the technical implementation can be found in Appendix B.

\subsection{Experimental Setup and Task}

In the 4A task, the human used his two biological arms to work on an object, while being assisted by two robotic arms for supporting and moving the object. The two robotic arms were composed of two KUKA LWR IV+ robots. Each robot was equipped with joint torque sensors at the actuators and were torque-controlled. A 6-axis ATI-force torque sensor was mounted on each end-effector on which a $3 \mathrm{D}$ printed flat palm tool was attached for grasping. Two foot interfaces were used as master interfaces to share the control of the 


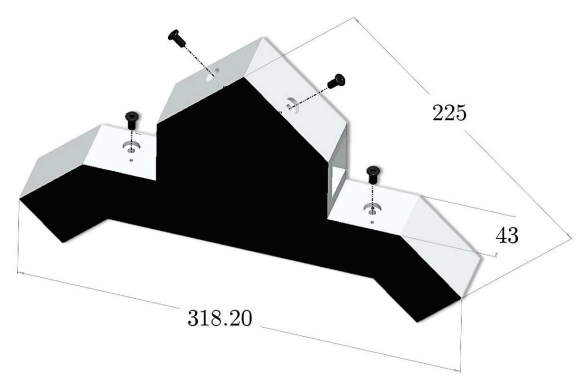

Figure 5. The object was a 3D printed piece with an arbitrary shape and with a mass of $0.525 \mathrm{~kg}$. Four thread inserts are present at different angles in which assembled M5 hexhead screws had to be checked and tightened/loosened where needed. The relevant dimensions of the object are present in the figure (units are in $\mathrm{mm}$ ).

robotic arms with the human. The object to manipulate is illustrated in Figure 5.

The 4A task consisted in reaching, grasping, and moving the object between different locations, in order to inspect and tighten/loosen the screws, and to weigh the object. The object was initially placed at a fixed position on a wooden board that served as a table (see Figure 6a). On one side of the table, the user sat on a chair with both feet positioned on the foot interfaces. The initial distance between the feet was approximately $0.5 \mathrm{~m}$. Each foot was placed in the center of each platform's workspace. We decided to not constrain the type of footwear, so users were free to use their own shoes. Moreover, the footrest of the platform was adjusted in length to fit the participant's shoes. Then their feet were strapped and secured to the platforms. Both platforms were horizontally aligned. The distance between the robot's bases was approximately $0.9 \mathrm{~m}$. The initial resting position of the robots' end-effectors was approximately at the center of the working table and at a height matching the level of the user's chest. The chair position was adjusted for the user to be able to always begin in a neutral position of $90^{\circ}$ angles in hip-knee-ankle (w.r.t. horizontal axis). However, the chair's height and the distance between the foot interfaces were kept fixed for all participants. If a participant did not meet approximately $90^{\circ}$ angle in the knee due to being tall, to help them meet this initial condition, extra cushions were used on the chair,. The two robotic arms were placed in front of the human on the other side of the table. From there, the user was asked to complete the 4A task by achieving the following sub-tasks successively:

1. Starting from their initial position (Figure 6a), control the robotic arms to reach the object and grasp it (Figure 6b). The grasp is encouraged by coordinating the position of the end-effectors as diametrically opposed. As a result, the holding force is enabled by the normal forces of each robotic arm on the object, as well as the friction they provoke by the interaction with gravity.

2. Lift the object and bring it in front of him (Figure 6c).

3. Use his hands to tighten/loosen the screws while the object is supported by the robot (Figure 6d). If possible, the human should use the robots to rotate the object for better access to the screws.

4. Bring and position the object on a balance (Figure 6e).

5. Break the grasp (Figure 6f) and weigh the object (Figure $6 g)$.

6. Reach and grasp the object again.

7. Move the object to the final position on the right (Figure $6 \mathrm{~h}$ ) and release the grasp (Figure 6i).

8. Bring the robots to their initial pose (Figure $6 \mathrm{j}$ ).

The above sub-tasks are illustrated in Figure 6 and demonstrated in Extension 1. Twelve subjects performed the 4A task four times, to test the four control strategies. All subjects were male lab members, $28.00 \pm 2.93$ years old and initially naive in the use of foot interfaces to control robotic arms. They were however familiar with robotic systems and/or work with robot technologies. The demonstrations of one participant performing the task with each control strategy can be found in Extensions 1, 2, 3, and 4. For each subject, the order of the control strategies was randomized. For each control strategy, each participant had a preliminary training session lasting up to 5 min (depending on the participant's confidence), to become familiar with the control mode, including the mapping, scaling, and haptic feedback from the foot interfaces.

The mapping according to the control strategy is explained in Figure 7 (Extension 5).

Regarding haptic control, for the axes using a positionvelocity mapping (i.e., yaw and roll), a joystick was simulated via a virtual spring-damper. Although axes were used for position-position mapping (i.e., $x, y$, pitch), damping was used to smooth down the motion of the feet. All these gains were experimentally tuned for the comfort of the user, based on an exploratory study of two people (both males, aged $26.5 \pm 0.5$ ), to be uniform across control strategies. Furthermore, the range of forces we allowed to reflect at the level of the feet was carefully chosen based on findings from related works for adequate perception and to avoid startling the user (Abbink and van der Helm (2004); 


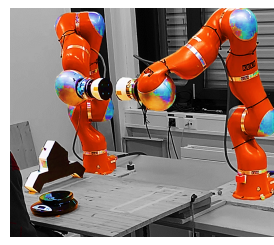

(a)

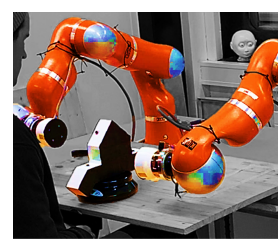

(f)

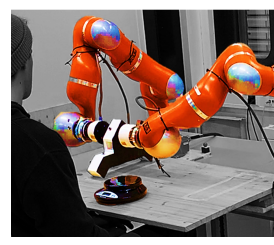

(b)

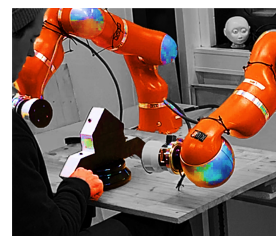

(g)

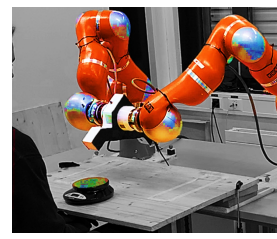

(c)

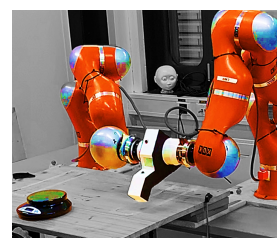

(h)

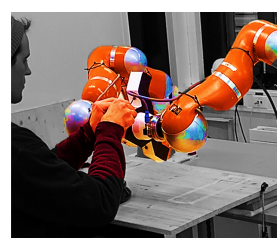

(d)

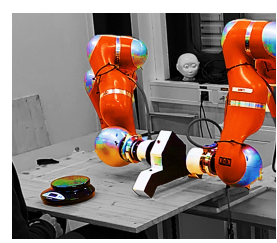

(i)

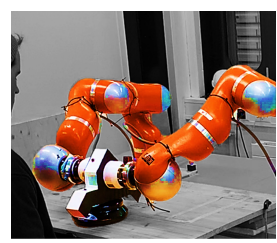

(e)

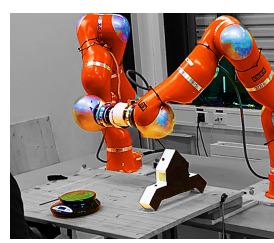

(j)

Figure 6. Snapshots of the four-arm manipulation task performed by a human using the $U+F$ control strategy. Starting from their initial resting position (6a), the human moves the robots to reach and grasp the object located at the initial position on his left. Once the grasp is secured (6b), the human lifts the object to bring it in front of him (6c) and starts tightening/loosening the screws on the object with his hands $(6 \mathrm{~d})$. In this phase, the human can control the robots in order to rotate the object to have a better access to the screws. After this, the human brings the object on a weight scale in front of him (6e). Once this is done, the human breaks the grasp (6f) and measures the object's weight (6g). Then, the object should be grasped again and transported to the final position on the right (6h). Finally, after breaking the grasp (6i), the human moves the robots to their initial position approximately (6j). For clarity, please check Extension 1.

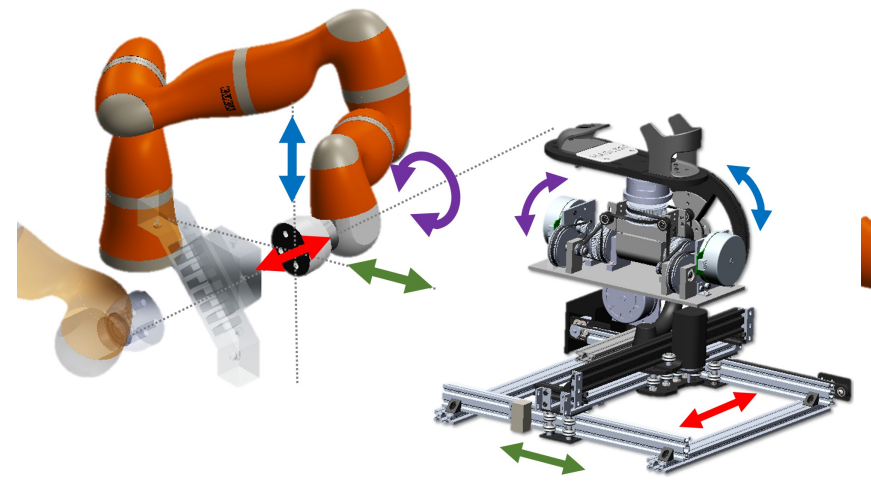

(a)

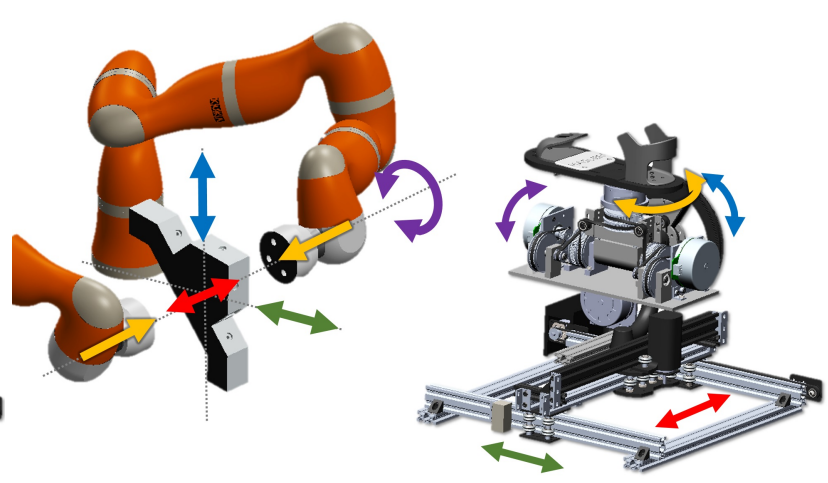

(b)

Figure 7. Color-code mapping of degrees of freedom between the 5-DoF foot platforms and the robotic arms. Figure 7a represents the uncoordinated strategies $(U$ and $U+F)$ where each foot controls their ipsilateral robotic arm (Figure 1). Figure 7b shows the mapping of the coordinated strategies $(C, C+F)$ where, once the object is grasped, one foot has the synergistic control of two robotic arms with respect to their center position. In both cases, the linear axes in the horizontal plane (red and green) are also mapped as linear axes, and the pitch tilt is transformed in the linear vertical ( $z$ ) motion (blue). Whereas, the roll motion (purple) is mapped in the self-rotation of the end-effector of the robotic arm. Note that the control strategies with coordination (Figure 7b) include the yaw rotation (yellow) for controlling the opening/closing of the coordinated grasp (linear motion). In general, a position-velocity mapping is used for roll and yaw, as this mapping is better when the required range of motion of the robot is large with respect to the human input (Kim et al. (1987)). Finally, the directions of the roll rotations in the uncoordinated control-strategies are made consistent with the coordinated strategies, by adopting a sagital plane of symmetry between both feet. Hence, when both feet do a roll in opposite directions (bio-mechanically the same muscle groups), the end-effectors rotate in the same direction. For clarity, please check Extension 5.

Ichinose et al. (2013); Geitner et al. (2018)).

After training, the participant was asked to perform the real task within $5 \min$. Figure 8 illustrates two of the experiments performed with one participant with the $U+F$ and $C+F$ control strategies. Grasping state, coordination state, and measured and desired robot contact-forces are depicted. The experimental protocol was approved by the Human Research Ethics Committee of EPFL, and the methods were carried out in accordance with the approved protocol. The procedure adhered to basic ethical practice from the Nuremberg Code.

\subsection{Evaluation Metrics}

To study the differences between the four control strategies, we defined the subjective and objective metrics. They include two questionnaires that were filled out for each control strategy: 

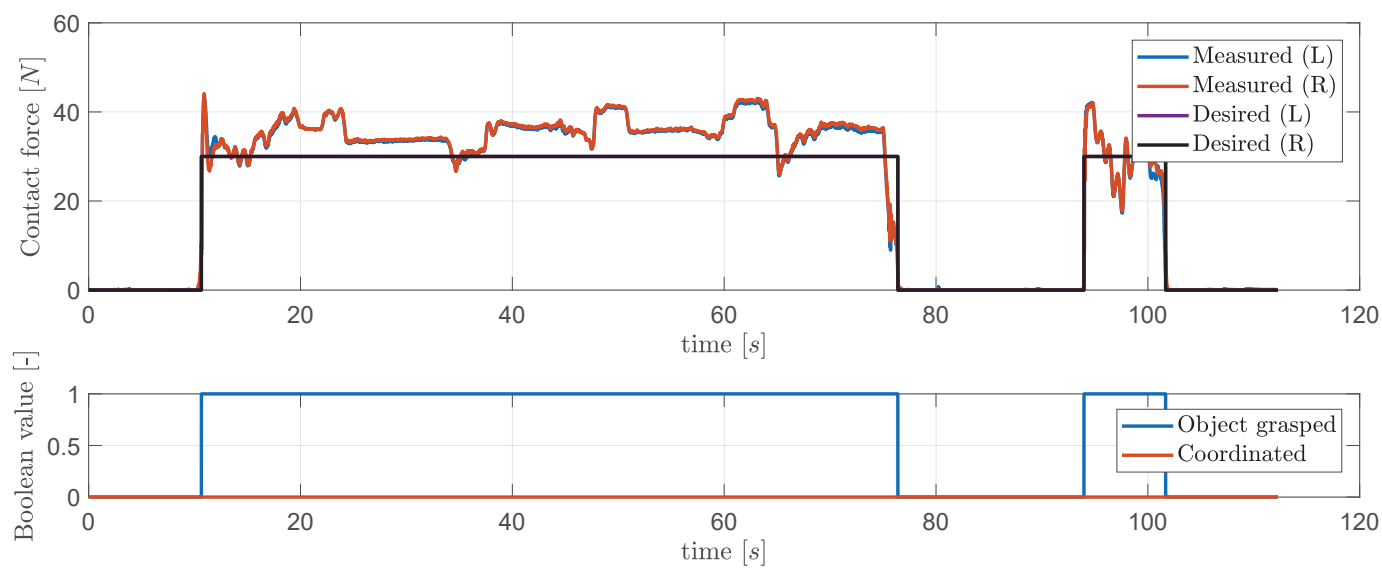

(a)
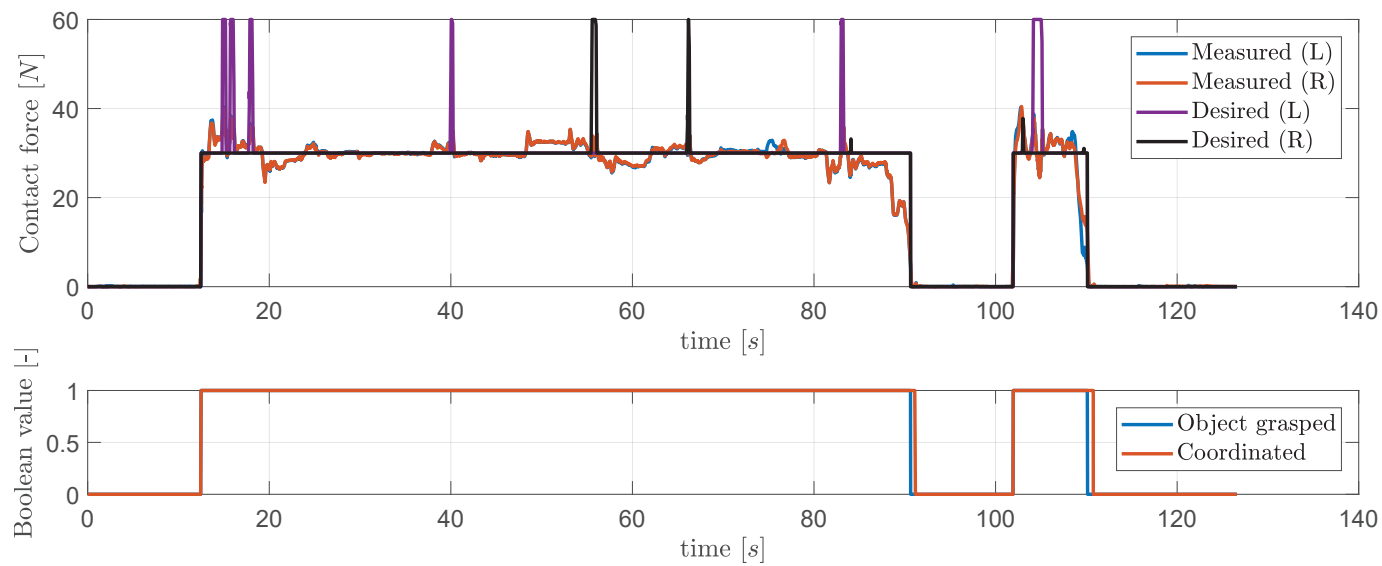

(b)

Figure 8. Examples of measured and desired interaction forces obtained with the shared control strategies including force assistance, namely $U+F(8 \mathrm{a})$, and $C+F(8 \mathrm{~b})$. The "object grasped" signal informs about the object's grasping state based on the grasp condition (12). The "coordinated" signal reflects whether the robot moves in coordinated manner or not. $(L)$ and $(R)$ refer to the left and right robots, respectively. Note that, for control strategy $U+F$, both robots have the same desired force profile when the object is grasped. For $C+F$, the desired force profiles are designed as explained in Appendix B. These examples are obtained from one participant of the user study.

- The first questionnaire measures perception of fluency of the interaction through eight categories: ease of use, usefulness, predictability, fluency, trust, goal perception, robot intelligence and satisfaction (Hoffman (2019)). Figure 9 shows the list of questions/items for each category with the associated Cronbach's alpha value as a measure of internal consistency.

- The second questionnaire is the NASA Task-Load Index (NASA-TLX) that measures the participant's performance demand (i.e., how satisfied is the user with their performance -in reversed scale-), effort (i.e., how hard -physically and mentally- the user has to work to accomplish their level of performance), frustration (i.e., how frustrated the user felt), mental demand (i.e., how much mental activity was required), physical demand (i.e., how much physical activity was required), and temporal demand (i.e., pace of the execution of the task) of the task (Hart and Staveland (1988)).
The answers to these questionnaires were assessed using a 5 Point Likert Scale from $1=$ strong disagreement to $5=$ strong agreement.

The objective metrics are composed by

- The individual sub-tasks' efficiency $\sigma_{k}$ defined as:

$$
\sigma_{k}=\frac{t_{\max }-t_{k}}{t_{\max }}
$$

where $\sigma_{k} \in[0,1]$ is the relative efficiency metric for the sub-task $k$, with $k \in\left[1, s_{\max }\right]$, where $s_{\max }=8$ is the maximum number of sub-tasks. This metric considers timeliness because $t_{k}$ is the time needed to perform subtask $k$, and $t_{\max }=5 \mathrm{~min}$ is the maximum time allowed for the overall task. Consequently, when a sub-task was not accomplished, $t_{k}$ is considered to be equal to $t_{\max }$, hence the efficiency $\left(\sigma_{k}\right)$ is zero. 
Ease of Use (Cronbach $\alpha=0.9481$ )

1. "Overall, it was easier to execute the 4Armed task (w.r.t the other modes)."

2. "Overall, it was more tiresome to execute the 4Armed task (w.r.t the other modes)" $\mathbf{R}$

3. "It was easier to move the robotic arms."

4. "It was easier to manipulate the object."

"It was easier to move the object."

"It was easier to rotate the object."

7. "Overall, I felt I was improving the performance as the trials progressed."

8. "This system was easy to use."

9. "This system was simple to use."

10. "This system was user friendly (easy to understand)."

11. "This system was flexible."

12. "This system was effortless."

13. "I could use this system without written instructions."

14. "I did not notice any inconsistencies as I used it."

15. "Both occasional and regular users would like it."

16. "I could recover from mistakes quickly and easily."

17. "I could use it successfully every time."

Usefulness

1. "Overall, this strategy was useful."

Predictability

1. "The robots responded to my motion inputs in a predictable way."

Fluency (Cronbach $\alpha=\mathbf{0 . 8 8 8 9}$ )

1. "The robots and I worked fluently together as a team."

2. "The robots contributed to the fluency of the interaction."

Trust (Cronbach $\alpha=\mathbf{0 . 8 4 6 2 )}$

1. "I trusted the robot to do the right thing at the right time."

2. "The robots were trustworthy."

Goal Perception

1. "The robots perceived accurately what my goals were."

Robot Intelligence (Cronbach $\boldsymbol{\alpha}=\mathbf{0 . 9 0 0 3 )}$

1. "The robots were intelligent."

2. "The robots were able to independently make decisions throughout the task."

3. "The robots had an understanding of the task."

4. "The robots had an understanding of my goal during the task."

Satisfaction (Cronbach $\alpha=0.9094$ )

1. "Overall, I preferred this strategy."

2. "I am satisfied with the $4 A$ system."

3. "This system was fun to use."

4. "This system worked the way I wanted it to work."

Figure 9. Subjective questionnaire to Evaluate Fluency of the Shared Control (Hoffman (2019)). R indicates reverse scale. Internal consistency in the categories featuring multiple questions is tested using Cronbach's Alpha. Note that there is high internal consistency as $\alpha>0.84$.

- The number of object falls $N_{f}$ that counts the number of times the object falls during the task (e.g., while being grasped or after collision with one of the robotic arms).

- The number of sub-tasks achieved $s \in\left[0, s_{\max }\right]$ measuring completeness of the whole 4A task.

- The overall success metric $\beta_{T}$ encompassing the subtasks' efficiency, the total number of sub-tasks to achieve and the number of object falls, which we define as

$$
\beta_{T}=\sum_{k}^{s} \sigma_{k} / s_{\max } \mathrm{e}^{\frac{N_{f}}{s_{\max }}}
$$

with $\beta_{T} \in[0,1]$. Note that replacing (26) in (27) yields

$$
\beta_{T}=\left(s-\frac{t_{\text {total }}}{t_{\max }}\right) / s_{\max } \mathrm{e}^{\frac{N_{f}}{s_{\max }}}
$$

where, $t_{\text {total }}$ is the total time used to complete the whole task. This formula considers efficiency and completeness, and it penalizes object falls.

Finally, an observation of the average forces and standard deviations in either robot arm is also made, as an intuition of which control strategy achieves the more robust and stable grasp.

\subsection{Statistical studies}

\subsubsection{Description}

Two statistical studies are conducted in this work. In Study I, we analyze the effect of the four different control strategies $(U, U+F, C+F$, and $C)$. In Study II, we analyze the individual effect of the assistance modalities: force assistance (F.A) and auto-coordination assistance (C.A), as well as their interaction. The responses measured correspond to the answers to the questions and objective metrics presented above in the protocol (Section 3.2).

\subsubsection{Hypotheses}

For Study I, our main hypothesis is that the $C+F$ control strategy will outperform all the others, in all subjective and objective metrics. Indeed, as force and coordination assistance are both used, we expect to observe better scores generally, especially in metrics such as mental demand, physical demand, number of object falls, or success.

Regarding Study II, several hypothesis can be drawn and are listed below:

- By controlling both robots with one foot and focusing only on the motion of the object, we first hypothesize that coordination assistance will reduce the task load. However, we do not expect temporal demand to be reduced, as the pace of the task is mainly decided by the user.

- We expect an increase in the perceived fluency and the downstream outcomes when coordination assistance is used.

- For the objective metrics, we hypothesize that coordination assistance will positively affect the sub-tasks' efficiency, particularly for those involving object motion and when self-rotating the object is needed during the four-arm screwing task. Coordination assistance should improve completeness and overall success. 
- However, we do not expect coordination assistance to reduce the number of falls that is maybe more related to grasping forces.

- By delegating the grasp responsibility to the robots, we hypothesize that force assistance will also significantly reduce the task load, except for the temporal demand.

- Therefore, we expect force assistance to positively affect the categories related to fluency.

- We also hypothesize force assistance to increase the efficiency of sub-tasks where the object is grasped.

- We expect force assistance to significantly reduce the number of falls and accordingly, positively affect completeness and overall success.

- Finally, in agreement with our hypothesis in Study I, we hypothesize that the interaction of force assistance, when combined with coordination assistance, will improve the results from all subjective and objective metrics.

\subsubsection{Analysis Procedure}

Data sets were analyzed for normality by using Jarque-Bera test. For normally distributed data in Study I, a one-way analysis of variance (ANOVA) with repeated measures was used for differences among the four control strategies in the responses of objective and subjective metrics explained in Section 3. To avoid type I error, the Tukey Honestly Significant Difference (HSD) was used, as a post-hoc analysis for multiple pairwise comparisons. Similarly, for non-normally distributed data, the Kruskal-Wallis test was used as a non-parametric analysis, as well as the Dunn-Sidak test for post-hoc multi-comparison. For Study II, a n-Way ANOVA was used to test statistical significance among assistance modalities (F.A,C.A) and to consider their interactions ( $F . A * C . A$ ). These main effects and interaction effects are showcased as a constant coefficients model for the normally distributed data. The effect of each factor and their interactions is presented as a dot plot per each response. Whereas, a Friedman non-parametric test was used to analyze the differences between assistance modalities. As this test computes the effect of only one treatment at a time (column effects adjusted for the row effects), it was performed twice, once per each mode of assistance ( $F . A$ or $C . A$ ), and participants' trials were considered as repetitions. Note that the Friedman test does not test for probabilities of interaction effects. All these tests were performed with a significance tolerance of $<5 \%$.

The constant coefficients model of the n-way Analysis of variance is described as

$$
y_{m n o}=\kappa+\gamma_{m}^{C . A}+\gamma_{n}^{F . A}+\gamma_{m n}^{C . A * F \cdot A}+\epsilon_{m n o}
$$

Where $y_{m n o}$ represents the response variable, specifically our subjective or objective metrics of the task, of observation number $o . \gamma_{m}^{C . A}$ represents the $m^{\text {th }}$ coefficient of the two levels or groups of the coordination assistance factor $(m$ = 1: uncoordinated, $U, m=2$ : coordinated, $C$ ), and $\gamma_{n}^{F . A}$ is the $n^{\text {th }}$ coefficient of the two levels or groups of the force assistance factor ( $n=1$ : without force assistance, wo.F. $n=2$ : with force assistance, w.F). Similarly, $\gamma_{m n}^{C . A * F . A}$ represents the interaction coefficient for the $m^{\text {th }}$ group of coordination assistance with the $n^{\text {th }}$ group of force assistance. Finally $\kappa$ represents the constant or overall mean, whereas $\epsilon$ represents the residuals of the model, assumed to be normally distributed, independent and with constant variance (homoscedasticity). The coefficients represent deviations of the factor's group from the overall mean $\kappa$, so that

$$
\left\{\begin{array}{l}
\Sigma_{m=1}^{M} \gamma_{m}^{C A}=0 \\
\Sigma_{n=1}^{N} \gamma_{n}^{F A}=0 \\
\Sigma_{m=1}^{M} \Sigma_{n=1}^{N} \gamma_{m n}^{C A * F A}=0
\end{array}\right.
$$

where $M=2$ and $N=2$ are the maximum number of levels per grouping factor.

For this reason, given only two levels or groups per qualitative main effect (assistance modality), the coefficients among each group have the same magnitude with opposite signs. Likewise for the interaction effects, two coefficients have the same magnitude as the other two, but with opposite signs (see Figure 13).

\section{Results}

\subsection{Study l: Response Differences among Control Strategies $(U, U+F, C+F, C)$}

Results of the subjective and objective metrics of Study I can be observed in Figures 10 and 11, respectively.

\subsubsection{Subjective Metrics (Task-load Index and Subjective Fluency of the Shared Control)}

For these questionnaires, all answers were normally distributed, hence parametric tests were used for analysis. Figure 10 shows the results of these subjective metrics.

\section{S-1. Task Load}

Results in task-load index reveal a significant difference 

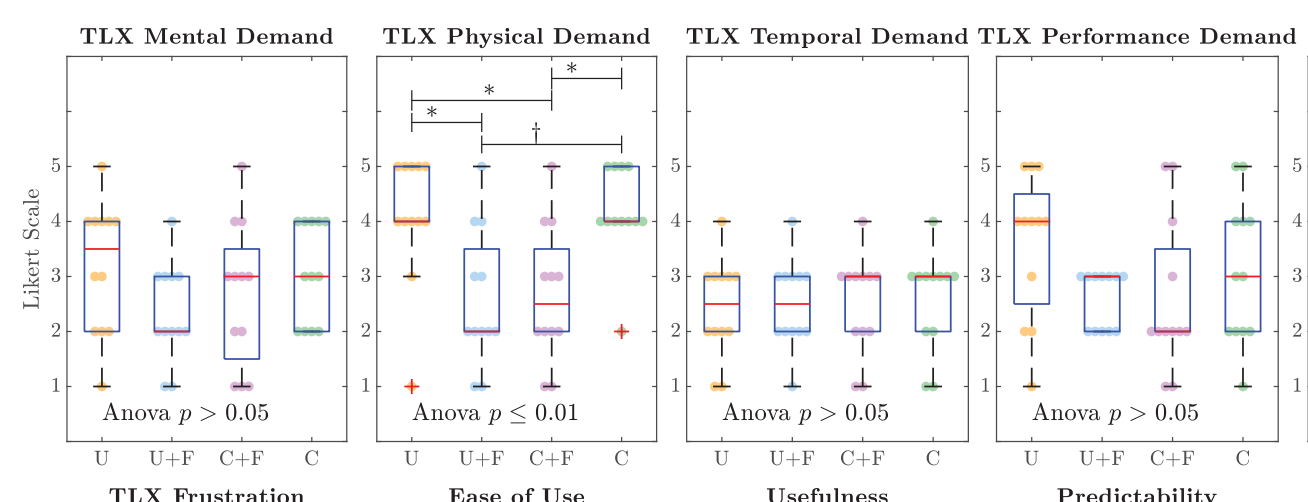

TLX Effort
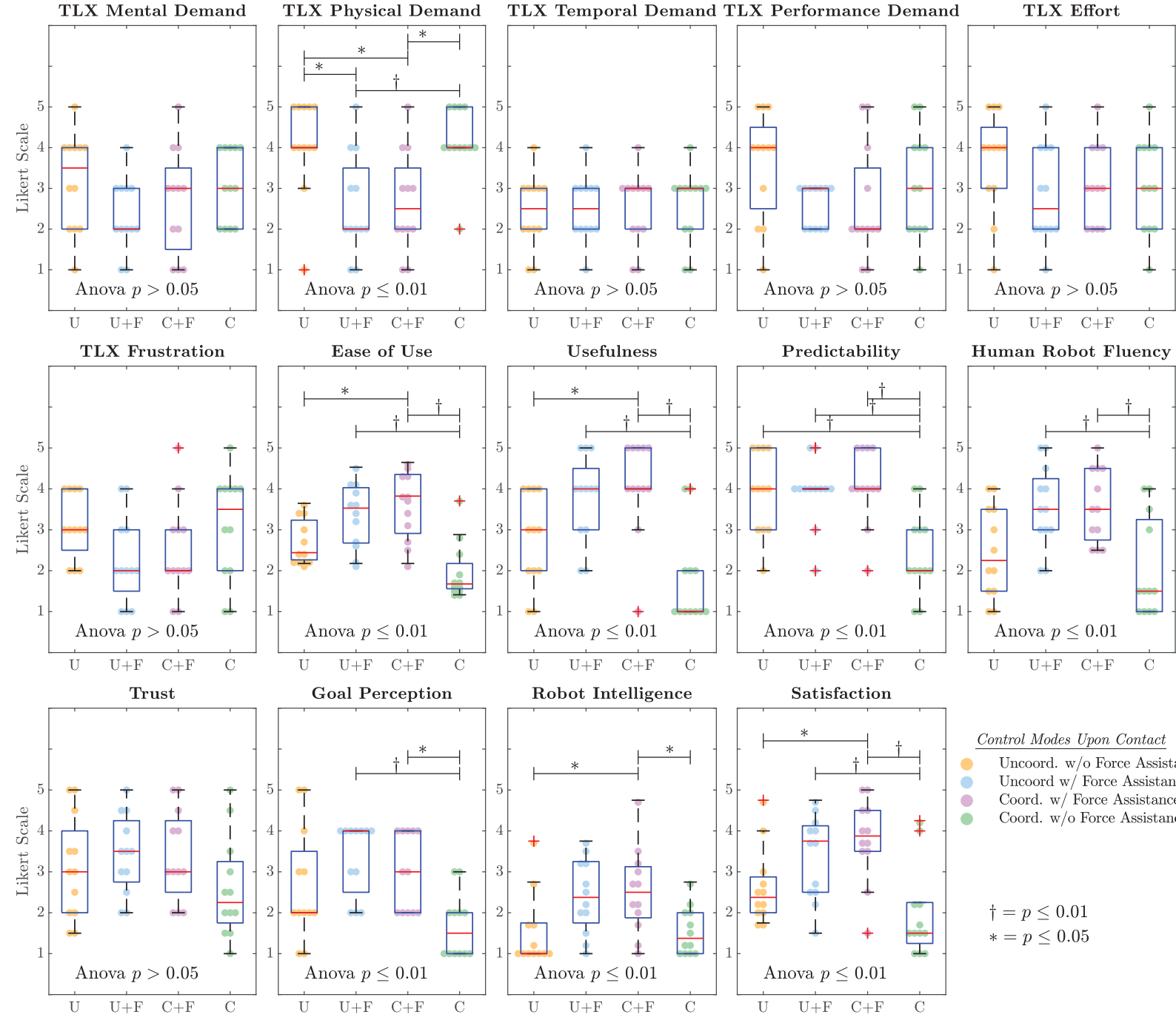

Control Modes Upon Contact

Uncoord. w/o Force Assistance Uncoord w/ Force Assistance Coord. w/ Force Assistance Coord. w/o Force Assistance

$\dagger=p \leq 0.01$

$*=p \leq 0.05$

Figure 10. NASA Task Load Index (the lower the better) and human-robot fluency (the higher the better) and outcomes of the shared control for a four-arm manipulation. Twelve people participated in this study. The Tukey plots are overlaid on the data points for clarity. According to the normality of the data, the type of statistical test (one-Way ANOVA with repeated measures or KruskalWallis) is indicated in each subplot, along with their p-value. Additionally, the pair-wise significant differences under $1 \%$ and $5 \%$ are indicated among the four sample means where applicable, with the symbols explained in the legend of the figure.

in physical demand among the four control strategies $\left(F=7.0722, p=5.57 \times 10^{-4}\right)$. The post-hoc HSD analysis shows that the control strategies with the lowest physical demand are those that include force assistance $U+F: 2.58 \pm$ 1.24 and $C+F: 2.66 \pm 1.23$ versus $U: 4.08 \pm 1.16, C$ : $4.16 \pm 0.83$. Furthermore, there are no statistical differences regarding the other elements of task load such as mental demand, performance, effort to improve, and frustration among the control strategies under trial.

\section{S-1. Fluency and Outcome Perception}

Similarly, there are significant differences of perception of ease of use $\left(F=12.6324, p=4.35 \times 10^{-6}\right)$ and usefulness $\left(F=10.5389, p=2.40 \times 10^{-5}\right)$. In these responses, control strategies with force assistance ranked higher than control strategy $C$. Specifically, for ease of use, the results of the post-hoc multi-comparison are $\{U+F: 3.37 \pm 0.78$, $C+F: 3.61 \pm 0.83\}$ versus $C: 1.97 \pm 0.69$, and for usefulness: $\quad\{U+F: 3.75 \pm 1.06, \quad C+F: 4.08 \pm 1.16\}$ versus $C: 1.75 \pm 1.14$.

Having both layers of shared control (force and coordination) is perceived as easier to use and more useful than non-aided telemanipulation $\left(p_{(C+F \text { vs } U)}=0.018\right.$ for ease of use and 

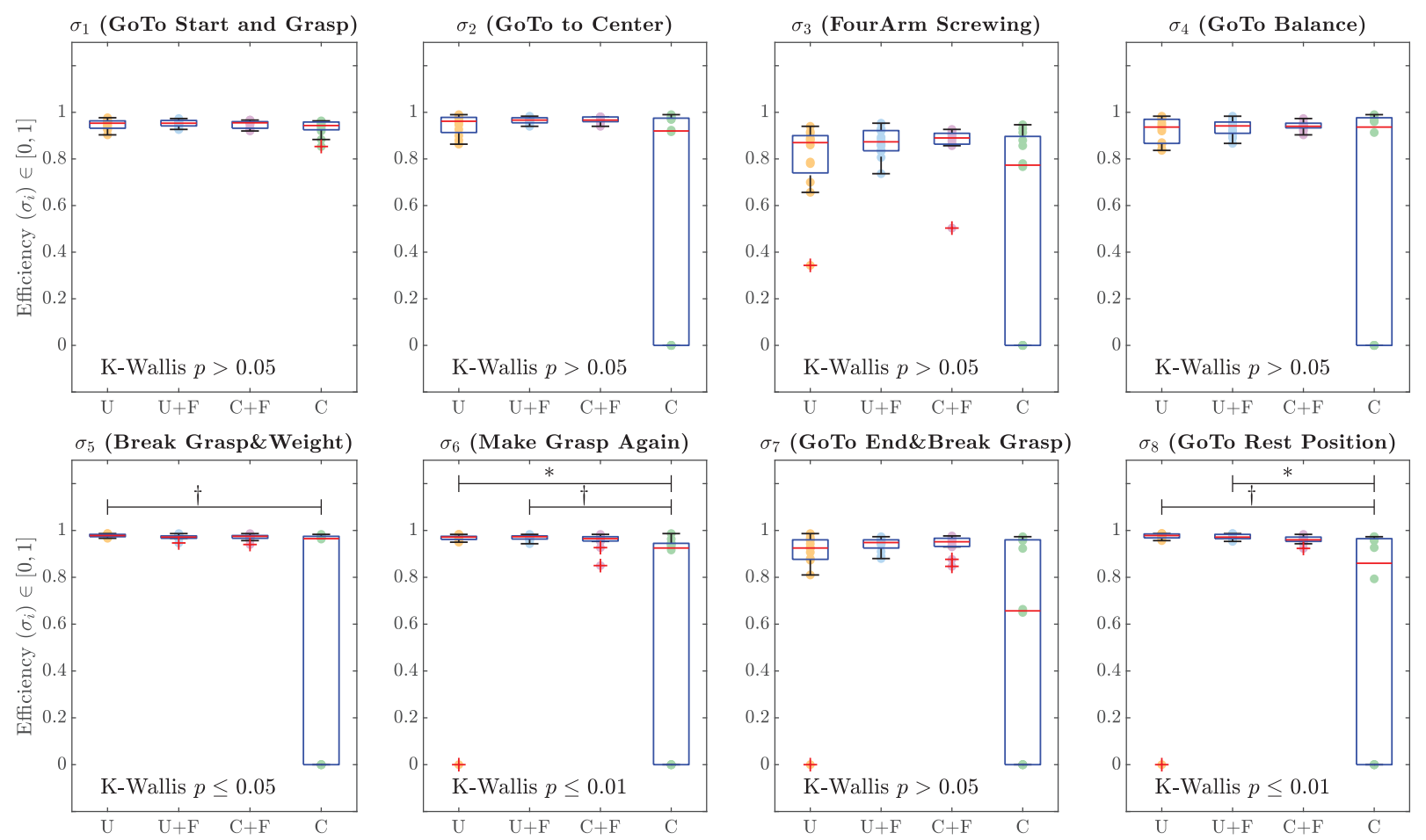

$\sigma_{7}$ (GoTo End\&Break Grasp)
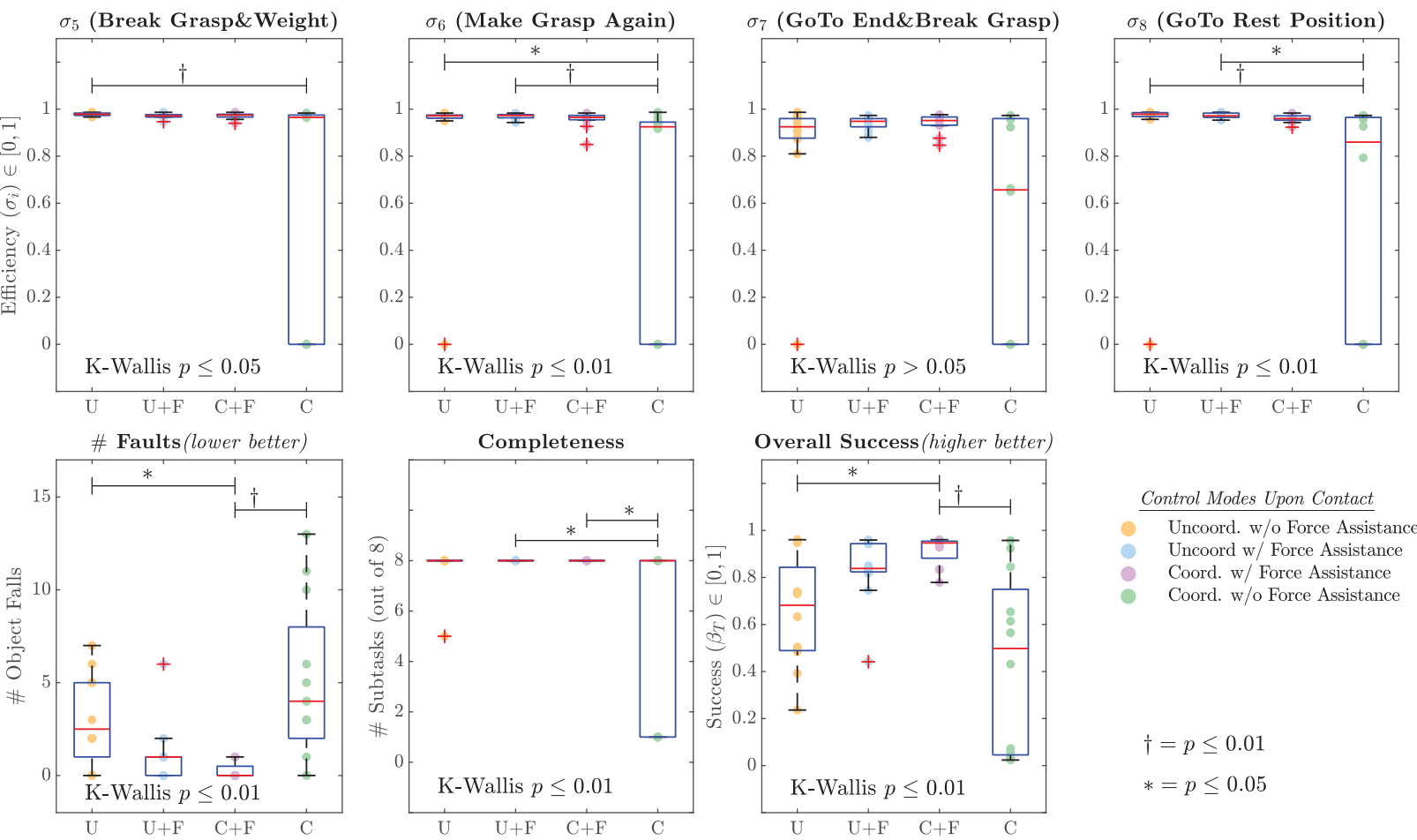

$\dagger=p \leq 0.01$

$*=p \leq 0.05$

Figure 11. Objective performance metrics. Relative efficiency of each sub-task (the higher the better), number of object falls (the lower the better), completeness and overall Success of the shared four-arm manipulation (the higher the better). Twelve people participated in this study. The Tukey plots are overlaid on the data points for clarity. According to the normality of the data, the type of statistical test (one-Way ANOVA with repeated measures or Kruskal-Wallis) is indicated in each subplot along with their $p$-value. Additionally, the pair-wise significant differences under $1 \%$ and $5 \%$ are indicated among the four sample means where applicable, with the symbols explained in the legend of the figure.

$p_{(C+F \text { vs } U)}=0.028$ for usefulness). Nonetheless, when adding force assistance, there is no significant improvement in user perception of ease of use and usefulness for uncoordinated control (ease of use $p_{(U+F \text { vs } U)}=0.120$ and usefulness $\left.p_{(U+F \text { vs } U)}=0.1454\right)$.

There is a clear difference in terms of predictability $\left(F=8.5664, \quad p=1.363 \times 10^{-4}\right)$ for control strategy $C$ versus all the other strategies. The former having the lowest predictability ( $2.42 \pm 1.00$ out of five).

Human-robot fluency also reports significant differences $(F=6.3591, p=0.0011)$ : Control strategies with force assistance were found as achieving more fluent interactions, at least against the control strategy $C\left(p_{(U+F \text { vs } C)}=0.0067\right.$ and $\left.p_{(C+F \text { vs } C)}=0.0051\right)$. A similar tendency is found in the subjective evaluation of goal perception $(F=5.9517$, $p=0.0017)$, where the lack of force assistance in control strategy $C$ affects negatively how the human regards the robot's understanding of the task $(C: 1.67 \pm 0.78$ out of five). Not surprisingly, significant differences were obtained in perception of robot intelligence $(F=4.7204$, $p=0.0061$ ), where control strategy $C+F$ was ranked higher and significantly different with respect to the control strategies without force assistance $\left(p_{(C+F \text { vs } U)}=0.030\right.$ and $\left.p_{(C+F \text { vs } C)}=0.040\right)$. Finally, users found more overall satisfaction $\left(F=7.6983, \quad p=3.06 \times 10^{-4}\right) \quad$ in control strategies with force assistance versus those without. 

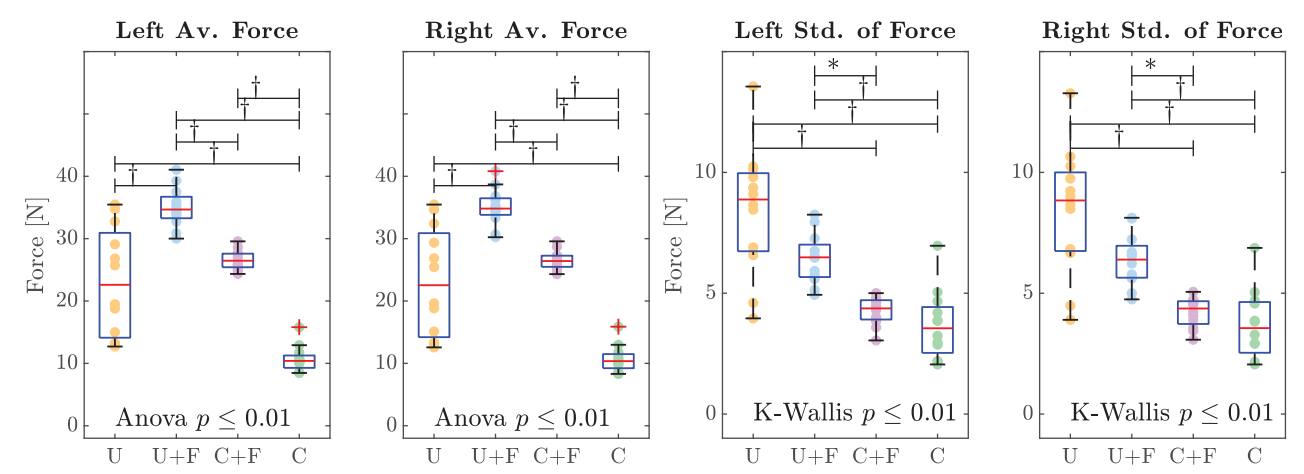

Control Modes Upon Contact Uncoord. w/o Force Assistance Uncoord w/ Force Assistance Coord. w/ Force Assistance Coord. w/o Force Assistance

$\dagger=p \leq 0.01$

$*=p \leq 0.05$

Figure 12. Averages and standard deviations of the forces (left and right robot) among the control strategies. Twelve people participated in this study. The Tukey plots are overlaid on the data points for clarity. According to the normality of the data, the type of statistical test (one-Way ANOVA with repeated measures or Kruskal-Wallis) is indicated in each subplot, along with their $\mathrm{p}$-value. Additionally, the pair-wise significant differences under $1 \%$ and $5 \%$ are indicated among the four sample means where applicable, with the symbols explained in the legend of the figure.

\subsubsection{Objective Metrics (Efficiency of Sub-tasks, Number of Object Falls, Completeness and Overall Success)}

Results from objective metrics were non-normally distributed. Hence, non-parametric statistical tools were used. Figure 11 shows the results obtained. Regarding the efficiency of sub-tasks, please note that when a sub-task was not completed due to difficulty or elapsed maximum time, the efficiency was estimated as zero.

\section{S-1. Efficiency of Sub-tasks}

For most sub-tasks (1. to 4. and 7.), all control strategies yield a (statistically) equal efficiency of median $84.02 \%$. However, this is not the case for releasing the grasp in the balance, i.e., sub-task 5. ( $\left.\chi^{2}=10.4779, p=0.0150\right)$ and regrasping, i.e, sub-task 6. $\left(\chi^{2}=14.1231, p=0.0 .0027\right)$, as well as going to rest position, i.e., sub-task $8 .\left(\chi^{2}=14.7519\right.$, $p=0.0 .0020$ ). When releasing the grasp in the balance (i.e., sub-task 5.), there is significantly lower efficiency for control strategy $C$ coordinated with respect to control strategy $U$. It is possible to observe an efficiency decrease from $U$ : $97.83 \%$ median to $C: 96.50 \%$, with a confidence value $p=0.0078$. Similarly, when re-grasping (i.e., sub-task 6.), there are significant differences between the strategies without coordination assistance and control strategy $C$. Specifically, the median efficiency for sub-task 6. drops from $U$ : $97.16 \%$ and $U+F: 97.33 \%$ to $C: 92.50 \%$ with p-values of $p_{(U \text { vs } C)}=0.0142$ and $p_{(U+F \text { vs } C)}=0.0037$. In the same way, when analysing sub-task 8 ., there are efficiency differences between $U, U+F$, and $C$. In this case, the p-values are $p_{(U \text { vs } C)}=0.0050$ and $p_{(U+F \text { vs } C)}=0.0116$, respectively.

\section{S-1. Number of Object Falls}

As expected, there are differences in object falls among the control strategies $\left(\chi^{2}=17.2258, p=6.35 \times 10^{-4}\right)$. In particular, control strategy $C+F$ reports a number of falls significantly lower than the control strategies without force assistance (i.e., $U$ and $C$ ). In this case, the median number of falls decreases from $U: 2.5$ and $C: 4$ to $C+F: 0$, with p-values $p_{(C+F \text { vs } U)}=0.0123$ and $p_{(C+F \text { vs } C)}=9.37 \times 10^{-4}$.

\section{S-1. Completeness}

In general, people managed to fully complete the eight sub-tasks 42 out of the total 48 runs of this experiment. Clearly, different control-strategies yield different levels of the completeness of sub-tasks $\left(\chi^{2}=13.2875, p=0.0041\right)$. Indeed, having only coordination assistance $(C)$ results in a lower number of sub-tasks completed with respect to control strategies with force assistance with significance values of $p_{(U+F \text { vs } C)}=0.011$ and $p_{(C+F \text { vs } C)}=0.011$.

\section{$S$-1. Overall Success}

There are differences in overall success among control strategies $\left(\chi^{2}=16.2135, p=0.001\right)$. As expected, control strategy $C+F$ outperforms control strategies without force assistance. In this case, median success rates improve from $U: 68.2 \%$ and $C: 49.8 \%$ to $C+F: 94.7 \%$. This with pvalues of $p_{(C+F \text { vs } U)}=0.04$ and $p_{(C+F \text { vs } C)}=9.00 \times 10^{-4}$. Interestingly, there is no significant lower success when only force assistance (i.e., $C+F$ vs $U+F$ ) is used.

\section{S-1. Average Force and Standard Deviation}

Figure 12 illustrates the results, in both robot arms, of statistical comparison in force average and standard deviation for the overall task among control strategies . Indeed, there were statistical differences in average force 

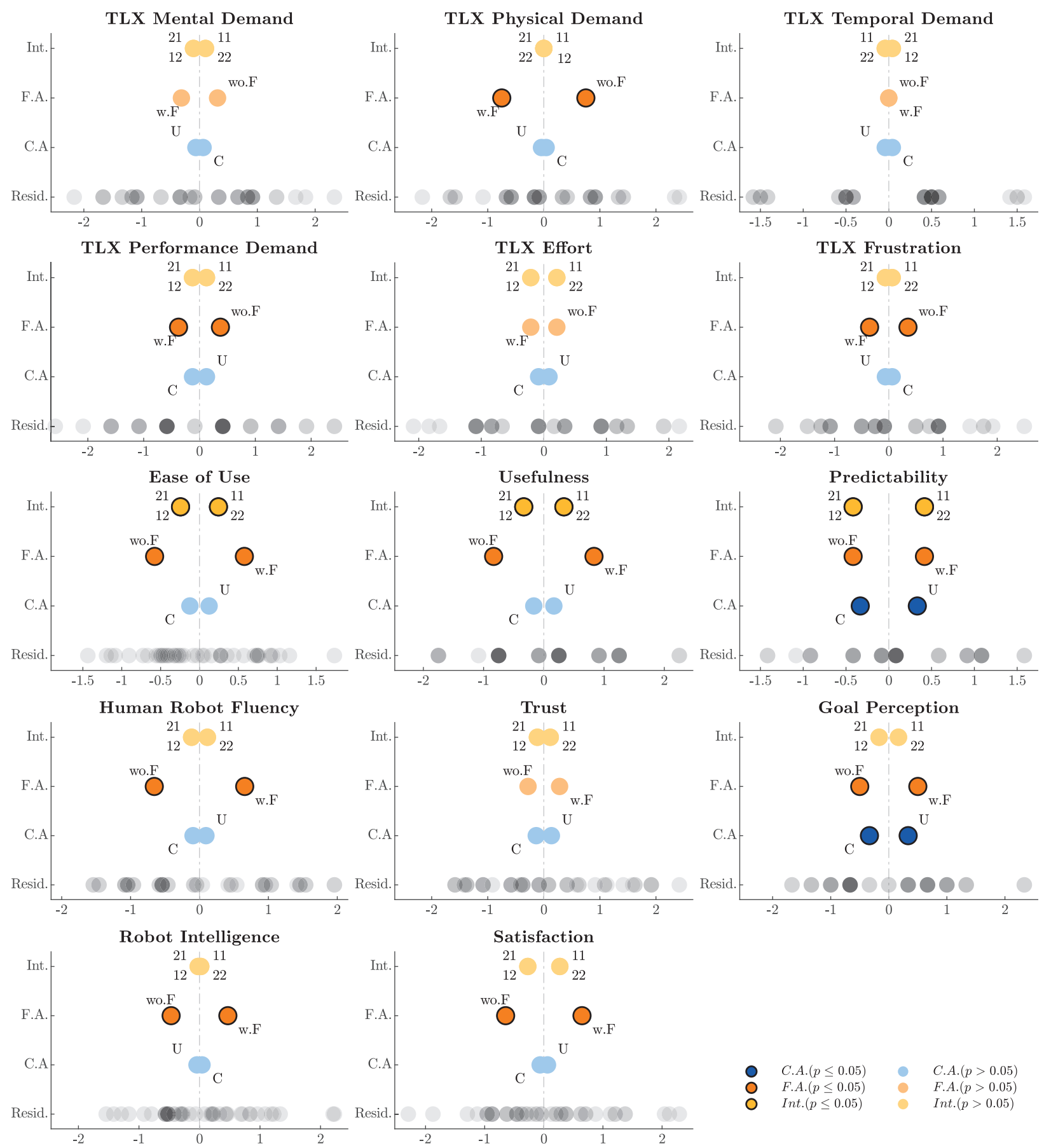

Figure 13. Dot-plots of the constant coefficient models for the subjective metrics of task load index and shared control fluency and outcomes. This plot enables us to observe the relative effect of the presence/absence of coordination $(C$. $A$ ) and force assistance $(F . A)$, as well as of their interaction. Statistically significant effects have a bold color and black edge. Residuals are faded and shown in first line of the dot-plots for comparison.

among the control modes, with $F=52.3711, p=1.45 \times$ $10^{-14}$ for the left robot, and $F=53.6479, p=9.57 \times 10^{-15}$ for the right robot. There were also differences among standard deviations with $\chi^{2}=29.2713, p=1.96 \times 10^{-6}$ for the left robot, and $\chi^{2}=28.3274, p=3.10 \times 10^{-6}$ for the right robot. The grasp of control strategy $C+F$ achieves the second highest strongest grasp (right: $26.64 \pm 1.59 \mathrm{~N}$, left: $26.57 \pm 1.54 \mathrm{~N}$ ) and maintains the second lowest median of standard deviations (left and right: $4.37 \mathrm{~N}$ ).
This could be the reason for the encouraging results of this control strategy with zero median object falls hence greater overall success. In contrast, in control strategy $C$, the average contact force applied by the participants is the lowest (right robot: $26.64 \pm 1.59 \mathrm{~N}$, left robot: $10.70 \pm 2.08$ $N$ ), and probably not sufficient to maintain a robust grasp, as reflected in the high amount of object falls. Moreover, only coordination assistance resulted in the lowest median standard deviation along the task $(C: 3.55 \mathrm{~N}$ for both 


$$
\eta(p \leq 0.05, \lambda=-1) \quad \eta(p=1, \lambda=0) \quad \eta(p \leq 0.05, \lambda=+1)
$$

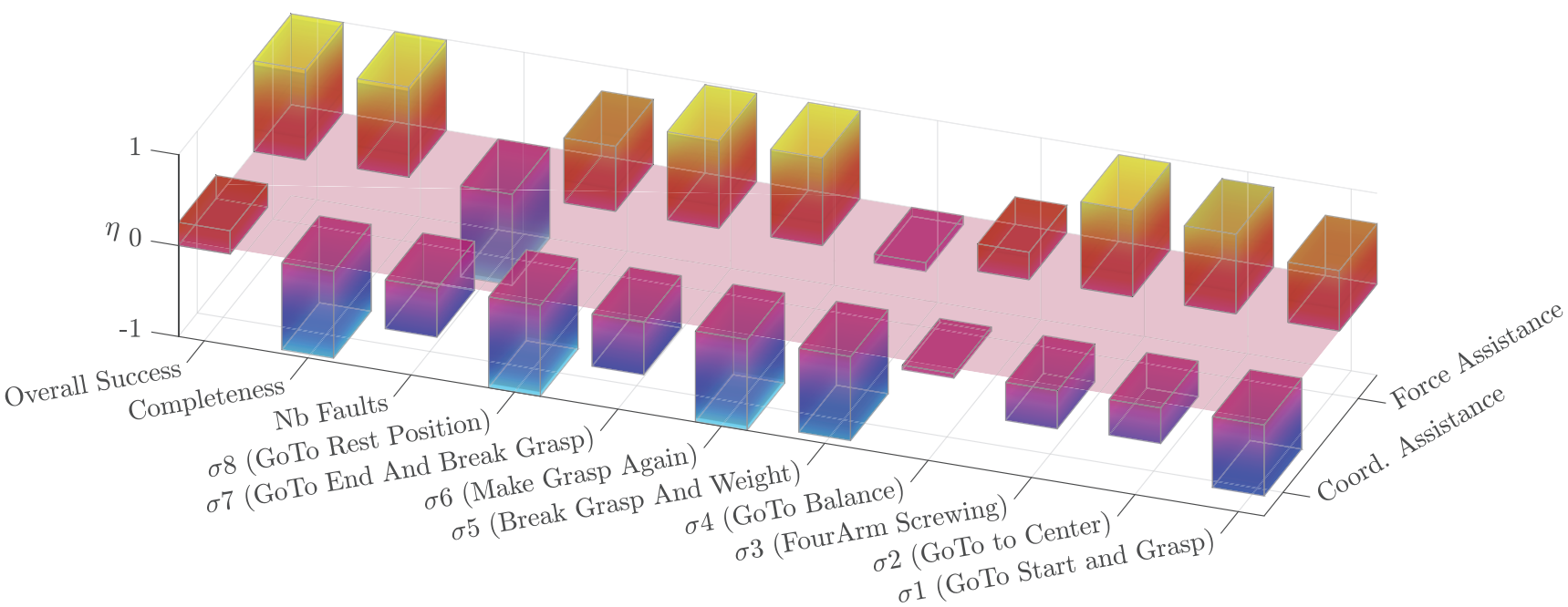

Figure 14. 3D Bar-plot to illustrate the relevance of each assistance modality in objective metrics of efficiency per sub-task, number of object falls, completeness and overall success. The results are based on a Friedman non-parametric analysis made twice, using each time as a column effect (factor) either coordination assistance or force assistance. The Friedman test does not enable us to test for interactions. Please note that the height of the bar does not represent the magnitude of the effect, as it is not modelled in the non-parametric analysis. Indeed, the height of the bar represents the function $\eta=\lambda(1-p)$, where $p$ is the $\mathrm{p}$-value of the $\chi^{2}$ Test. Furthermore, $\lambda \in[-1,1]$ is an integer that represents the effect of the factor. Specifically $\lambda=1$ when the presence of the factor causes a positive effect on the result. Conversely, $\lambda=-1$ when the effect is negative, and $\lambda=0$ when neutral. This effect's sign comes from comparing the mean ranks (from the Friedman Test) associated with each factor's group so that if the mean rank of (presence of assistance) > (absence of assistance), then the effect is positive, and conversely. Finally, the extreme of the color bars is highlighted where the $p$-value is less or equal to 0.05 . For more specific details about which categories have $p$-values that are statistically significant, please refer to the subsection of results of objective metrics in Study II.

arms) due to the difficulty that many participants found when holding and maintaining a grasp with a single foot, without force assistance. Therefore, we can deduce that when force assistance was added to coordination assistance, participants mostly delegated applying the grasping force to the robots; consequently, $C+F$ had the second lowest standard deviation.

\subsection{Study II: Main Effects and Interactions of the Assistance Modalities (C.A, F.A)}

The results of Study II on subjective and objective metrics can be observed in Figures 13 and 14 respectively.

\subsubsection{Subjective Metrics}

\section{S-2. Task load index}

The implementation of a force-assistance modality clearly gave the users the perception of alleviating their physical demand $\left(F=21.151, \quad p_{\text {F.A }}=3.58 \times 10^{-5}\right)$, frustration $\left(F=4.991, p_{\text {F.A }}=0.031\right)$ and performance demand $\left(F=4.816, p_{\text {F.A }}=0.034\right)$.

\section{S-2. Fluency}

Similarly, the presence of force assistance positively affected the ease of use $\left(F=30.949, p_{\text {F.A }}=1.47 \times 10^{-6}\right)$, humanrobot fluency $\left(F=18.153, p_{\text {F.A }}=1.06 \times 10^{-4}\right)$, predictability $\left(F=9.7345, p_{\text {F.A }}=0.003\right)$, goal perception $(F=11.478$, $\left.p_{\text {F.A }}=0.0015\right)$, usefulness $\left(F=26.347, p_{\text {F.A }}=6.21 \times 10^{-6}\right)$ , robot intelligence $\left(F=14.023, p_{\text {F.A }}=5.21 \times 10^{-6}\right)$, and satisfaction $\left(F=19.486, p_{\text {F.A }}=6.49 \times 10^{-5}\right)$. Whereas, the presence of coordination assistance had a negative effect on the predictability $\left(F=6.230, p_{\text {C.A }}=0.0168\right)$ and goal perception $\left(F=5.101, p_{\text {C.A }}=0.029\right)$. Nevertheless, when considering the interaction, the effect was reversed to positive for predictability $\left(F=9.735, p_{\text {F.A }} *_{\text {C.A }}=0.003\right)$. Also, the interaction among assistance modalities holds a positive effect on the ease of use $\left(F=2.525, p_{\text {F.A }{ }^{*} \text { C.A }}=0.023\right)$ and usefulness $\left(F=4.216, p_{\text {F.A } *_{\text {C.A }}}=0.046\right)$.

\subsubsection{Objective Metrics}

For objective metrics, only the relative main effects could be tested, as the Friedman non-parametric test does not include interactions.

\section{S-2. Efficiency of Sub-tasks}

It is clear that coordination assistance affects negatively the 
efficiency of the sub-tasks. This is statistically significant for sub-task 6. (re-grasping, $\chi^{2}=8.6521, p_{\text {C.A }}=0.0033$ ) and 8 . (go to rest position, $\chi^{2}=12.0896, p_{\text {C.A }}=5.0703 \times 10^{-4}$ ).

Regarding the presence of force assistance, there is a significant benefit for efficiency on sub-task 3. (four-arm screwing, $\left.\chi^{2}=4.0227, p_{\mathrm{F} . \mathrm{A}}=0.0449\right)$. Similarly, there is benefit for sub-task 6. $\left(\chi^{2}=4.4867, p_{\text {F.A }}=0.0342\right)$, as well as 7. (go to end and release the grasp, $\chi^{2}=4.8007$, $\left.p_{\text {F.A }}=0.0284\right)$.

\section{S-2. Number of Object Falls, Completeness, and Overall Success)}

Coordination assistance has a significant negative effect on the completeness $\left(\chi^{2}=4.1067, p_{\text {F.A }}=0.0427\right)$, but not in number of object falls and overall success.

Whereas, force assistance has a positive effect on the number of object falls, $\left(\chi^{2}=14.0890, \quad p_{\text {F.A }}=1.7436 \times 10^{-4}\right)$, completeness $\left(\chi^{2}=7.0169, \quad p_{\text {F.A }}=0.0081\right)$ and overall success $\left(\chi^{2}=12.9095, p_{\text {F.A }}=3.2692 \times 10^{-4}\right)$.

\section{Summary and Discussion}

In this work, we designed (shared) control strategies for four-arm manipulation tasks that involve the two user biological arms and two robotic arms that are controlled through the feet with haptic foot-interfaces. To help the user in supporting and moving the manipulated object, two assistance modalities are proposed: autonomous-contact force generation and auto-coordination of the robotic arms. The contributions of our work are two-fold:

1. We designed four shared-control strategies for the proactive assistance of feet-telemanipulation in performing four-arm manipulation tasks. In our scenario, the hands are free to physically interact with the system and to perform high-dexterity tasks upon an object.

2. We conducted a user study to evaluate the proposed strategies, as well as the individual and mixed effects of coordination and force assistance, on a four-arm manipulation task (two robots are controlled with the feet). We measured perceived workload, subjective human-robot fluency and (custom) objective metrics such as task efficiency, completeness, and success.

To evaluate the benefits of force-assistance and coordinationassistance modalities, we implemented four (shared) control strategies. The control strategies used impedance-based teleoperation, under the dynamical system's framework. All of them included haptic feedback (i.e., the measured interaction forces or the measured force errors when the strategy includes force assistance), as it is crucial for telemanipulation (Boessenkool et al. (2012); Khurshid et al. (2016)) and aids bi-pedal coordination (Roelofsen et al. (2016)). The four control-strategies are summarized below:

1. $U$ : This strategy does not provide either coordination or force assistance, and is used as a baseline. Each robot's end-effector tracks an attractor obtained from the foot pose.

2. $U+F$ : This control strategy offers force assistance, once the object is grasped. The user must bring the robots in contact with the object. Upon contact, the robots implement the grasp with a predefined target force. However, the user is always aware of the physical interaction with the object, through haptic feedback of the force error, and decides when to release the grasp. As this assisting behaviour is not catalyzed directly by the user, rather from a sliding window of interaction forces, we refer to this type of strategy as contact-initiated control strategy. We hypothesized that force assistance would alleviate the physical burden of the task on the user's side, hence improving the efficiency of the task.

3. $C+F$ : This strategy provides coordination assistance, through synergistic control of the two robot arms by a single foot. The user controls the position and orientation of a proxy frame collocated with the robots' center position. As this control strategy includes both modalities (force + coordination) of assistance, we hypothesized that it would outperform all the others, thus reducing both mental and physical burden. Hence, we expected that it would improve task efficiency and success. Haptic feedback is also used to facilitate the user re-gaining control of both robots in the transition from coordinated to non-coordinated behaviour.

4. $C$ : Once the object is grasped, this strategy offers auto-coordination of the two robot arms, but no force assistance. The user remains hence is responsible for generating a robust grasp. Auto-coordination, however, eases the transport, as the object can be moved with a single foot.

These strategies were compared to each other through a user study with twelve subjects using a four-arm manipulation, in which the robots are tasked to assist the holding, grasping, and carrying of a load. Subjective and objective metrics were defined to assess the benefits of each control strategy. By using two-way statistical analyses, we found the individual 
effect of both assistance modalities. The following is a detailed discussion on the effect of assistance modalities (Study II) and a comparison with what was known from the literature. Moreover, we analyze the effect of the interaction between coordination and force assistance in our scenario.

\subsection{Effect of Coordination Assistance}

Our task entails four arms (two biological arms and two foot-controlled surrogate robotic arms). As such, this is a complex set-up that requires bi-pedal, bi-manual and inter-limb (arm-foot) coordination. Although a person can use their natural bi-manual coordination, part of the task required asynchronous control of the limbs, which can lead to neural interference (Swinnen and Wenderoth (2004)).

Leg and arm coordination was however expected to be more complex to achieve. As shown in Serrien and Swinnen (1997), synchronous control of non-homologous effectors (e.g., leg and hand) result in coordination (stability and frequency) more limited than homologous effectors. They also found that as legs have different biophysical characteristics than arms (hence different natural frequencies); this disrupts symmetry (when moving non-homologous effectors) and attenuates synchronization. In other words, it is easier to synchronize either two feet or two hands, than it is to synchronize one foot and one hand. Also, moving a foot and a hand at the same frequency (1:1) yields coordination less stable than multi-frequency motions (e.g., $2: 1,3: 2$ ). (Kelso and Jeka (1992); Serrien and Swinnen (1997)). Furthermore Serrien and Swinnen (1997) found that the arm normally leads the leg during non-homologous coordination (because the characteristic frequency of the arm is higher than that of the leg). Moreover, there is a tendency for preferred synchronization in the same direction between ipsilateral limbs (Baldissera et al. (1982); Kelso and Jeka (1992)). Specifically, during coordination the foot will prefer moving in the same direction of the hand that is on the same (lateral) side of the body.

Acknowledging the challenges described above, we designed a task of reduced complexity for a four-arm shared manipulation. We designed bilaterally isomorphic sub-tasks for the feet (holding, moving and rotating an object), and the hands have a sub-task of (finger-alternating) rotary motion (i.e., bi-manual screwing with an Allen key). Substasks for the feet and hands also require force-application. Furthermore, we did not impose synchronized movements between the hands and feet. Rather, we wanted to see if people would naturally prefer to rotate the object (with the feet) and simultaneously screw (with the hands) to gain time; and to see how the type of control strategy would influence this tendency (see remark \#1 in Extension 6).

Handling the object with the feet can be divided in a transport and a rotation phases. In the transport phase, feet move iso-directionally in the horizontal, longitudinal, and antero-posterior axes. In the rotation phase, the feet rotate symmetrically (w.r.t sagital plane) in opposite directions (w.r.t antero-posterior axis) (see Figure 7a).

Our coordination-assistance modality consisted in controlling two robots using a single foot, the dominant one. Following the dynamic pattern approach to explain coordination, we expected our strategy to alleviate the musculo-skeletal constraints (c.f., Swinnen and Wenderoth (2004)) involved in the transport sub-tasks. In our experiments we noticed a bias when users performed dual-foot coordination. As can be observed in remark \#2 in Extension 6, when using two feet, there is an important lag between both legs' motion during the left-right horizontal transport. Our observations concur with those of Serrien and Swinnen (1997) of a preference to move first the dominant leg during lower inter-limb coordination, due to a strong functional asymmetry (stronger than in the upper-limbs).

This lag between legs was especially evident in the horizontal transport, but not as marked in the rotation phase and vertical transport. This is most probably due to the simultaneous side-by-side dual motion of the legs during the horizontal transport. This can be more destabilizing as it recruits non-homologous muscle groups (e.g., abductors vs adductors). This is in line with evidence that nonhomologous muscle activation produces coordination patterns less stable than homologous muscle activation (Swinnen et al. (1997)).

Consequently, we expected a single-foot modality to ease lateral object motion. Furthermore, we expected the spatio-temporal binding of the robots' motions to give a unifying experience of direct control of the object with the foot (evoking a gestalt phenomenon, c.f., Sanes and Truccolo (2003)). Such gestalt during grasping would decrease cognitive load and facilitate the remaining multilimb (i.e., two arms and one foot) coordination tasks.

\subsubsection{Subjective Task Load}

Perceived Mental Demand

Related works, such as Rakita et al. (2019) and Laghi et al. 
(2018), found that coordination assistance has a positive effect on the perceived mental demand for bi-manual tasks. However, our results did not confirm this hypothesis for our four-arm task. This could be due to the fact that our scenario uses the feet and that it is unusual to use a foot for manipulating objects. Indeed, grasping with a single foot, using abduction/adduction of the foot or yaw is a fairly unfamiliar gesture. In our task, the foot was used to control for both the grasping motion and grasping force. Results showed that using only a control strategy $C$ resulted in the weakest grip (see Figure 12) that had approximately half of the forces of control strategy $U$. People are used to grasping objects by using at least two end-effectors (e.g., two fingers, two hands, fingers against the palm, etc). Hence, perhaps grasping with a single foot was so unfamiliar (and felt not as robust as doing so with two feet) that this might have counterbalanced any benefit stemming from using a single foot in place of two.

Whereas, as can be observed in remark \#3 in Extension 6, subjects displayed a consistent tendency to continue moving the non-dominant foot, even after the auto-coordination is activated. And this behaviour recedes as the task evolves. This means that disrupting the bi-pedal coordination requires an extra mental effort to consciously stop the motion of the non-dominant foot. This pattern bifurcation from four to three limbs, causes the user to struggle in the switching phase. This, however, seems less relevant in the opposite sense (three-to-four switch).

\section{Perceived Physical Demand and Improvement Effort}

Our hypothesis was that coordination assistance would reduce perceived physical demand and effort, because of the expected decrease in physical stress (weight-bearing) of moving a single foot with respect to moving the two feet simultaneously. We expected similar results as in Laghi et al. (2018), but with more accentuated scores due to the heavier effectors (legs are heavier than arms). However, when using coordination assistance (Study II), we saw no significant differences in perceived physical relief and effort. As foot rotations are bio-mechanically coupled, they cannot be controlled completely independently. This is particularly relevant for the foot motion required to close the robots' grip and rotate the object, as yaw and roll of the foot are coupled in the talocrular joint (Siegler et al. (1988)). Due to this coupling, the range of motion of one rotation is affected by the other. But, this also affects the (postural) stiffness and damping of the ankle-joint, which are strongly dependent on the angular position and muscle activation, with a non-linear increase near the limits of motion (Hunter and Kearney (1982); Kearney et al. (1990)). Therefore, this requires a heavy toll for the internal foot rotation (yaw) that has the smallest range of motion $\left(16.29 \pm 3.88^{\circ}\right.$ Siegler et al. (1988)). Consequently, this makes it more difficult to simultaneously apply force, keep the foot position (to hold the grip) and move the object, when controlling for multiple rotations. Indeed, this extra physical effort counterbalances any potential physical relief when using one foot instead of two.

\subsubsection{Fluency}

We expected to see an increase in subjects' subjective perception of fluency with our coordination assistance modality, thereby concurring with related work (Rakita et al. (2019)).

For the scores of ease of use, usefulness, human-robot fluency, and trust, the coordination assistance did not provoke a significant difference in the participants' responses. However, regarding predictability and goal perception, contrary to what was expected, there was a significant negative effect when coordination assistance modality was used. For these reasons, our hypothesis was not validated. The reason for these unexpected results could be inline with these discussed previously for mental and physical demands. Specifically, the added difficulty of using yaw to keep the grasp with a single foot might have out-weighted the benefits brought by the fluency of the robots' joint motion resulting from the coordination assistance.

\subsubsection{Objective Metrics}

Efficiency of Sub-tasks

We hypothesized that coordination assistance would increase sub-task efficiency. We expected to see a positive effect on efficiency, particularly in sub-tasks involving moving the object around when grasped (i.e., sub-tasks 2., 4., and, 7.). Furthermore, in sub-task 3 . where the four arms worked in coordination to screw, the efficiency could have increased, because controlling the position of the object directly with one foot should have relieved the cognitive workload, making the rotation of the object simpler. Nonetheless, these hypotheses are rejected. Results showed that most of the sub-tasks (2., 3., 4., and 7.) were performed with no statistical differences in efficiency among the control strategies.

For sub-tasks where the object is not grasped (i.e., sub-tasks 1., 6., and 8.), we did not expect statistical differences as 
the coordination assistance was only activated upon contact. This is indeed what we observed for sub-task 1 ..

Regarding the sub-tasks 6. (i.e, re-grasping) and 8. (i.e., returning to rest position), the low time- efficiencies could be due to the extra time the user needs to move their feet to match the robots' positions when switching from coordinated to uncoordinated control (specifically, after the grasp was released). Note that having only coordination assistance represented a challenge for some participants (five out of twelve). Starting from sub-task 2., we observed a loss of stamina because maintaining the grasp was difficult, explaining the low efficiencies for control strategy $C$ in Figure 11.

\section{Number of Object Falls}

Our null hypothesis was that coordination assistance does not affect the number of object falls, as this is more related to the grasp robustness (force-related). Our results showed that indeed there was no significant effect of coordination assistance.

\section{Completeness and Overall Success}

We hypothesized that coordination assistance would have a positive effect on the completeness and overall success. Unfortunately, Study II showed that coordination negatively affects completeness and has no significant influence on the success rate of the task, hence our hypothesis is rejected.

\subsection{Effect of Force Assistance}

Force assistance for manipulating objects has been proven beneficial in several works such as in Griffin et al. (2005). Here we explore the effect in our user study. A demonstration highlighting the importance of force assistance can be appreciated in Extension 7.

\subsubsection{Subjective Task Load}

\section{Perceived Mental Demand}

We hypothesized an improvement (lower scores) on the subjects' perception of mental load. We thought that delegating the force application to the robot could alleviate the sensory-motor constraints involved in neural processing during multi-limb coordination (Swinnen and Wenderoth (2004)). In other words, we assumed that by autonomously generating grasping forces to hold the object, the user could better focus on the execution of the task (e.g., working on the object and moving it) and relieve their muscles. Nevertheless, the evidence was not enough to prove our hypothesis and it is rejected. Despite being positive, the effect of force assistance in TLX Mental Demand was not statistically significant. Perhaps the task was not challenging enough to drastically increase the mental demand when no force assistance was provided.

Perceived Physical Demand, Performance Demand and Frustration

Our hypothesis was that using force assistance would physically alleviate the task for the user. We also assumed a lower demand in performance and less frustration. Our hypotheses are correct. This was partially observed in Study I where control strategies with force assistance contributed to lower physical demands, performance demands and frustration with the task. The constant coefficient models from Study II further revealed that force assistance can influence as much $22.22 \%$ score reduction (with respect to the bias in the likert scale) for perceived physical demand, $12.68 \%$ reduction in performance demand and $12.98 \%$ reduction in frustration. These percentages were calculated using the constant coefficients of the force assistance divided by the bias of the model.

\section{Improvement Effort}

Similarly to the other elements of task load, we assumed that force assistance would make the user avoid working hard to achieve a good level of performance (namely TLX effort). Nevertheless, our hypothesis is rejected. Study II revealed that, although force assistance tended to decrease perceived TLX effort $(6.58 \%$ reduction w.r.t. the bias in the likert scale), the effect was not statistically significant.

\section{Temporal Demand}

We expected no change in temporal demand, as we assumed the force assistance would not influence the pressure felt due to the pace of the task. As the user was always the main actor of the task, they decided the speed of use of the control strategies. Results are in line with this hypothesis.

\subsubsection{Fluency}

We expected our shared control strategies to lead to higher scores in the categories related to perceived fluency and downstream outcomes.

Ease of Use, Usefulness, Predictability, Human-Robot Fluency, Goal Perception, Robot Intelligence and Satisfaction In these categories our hypothesis prevailed. Indeed, there was a general positive effect from the force assistance (i.e., higher scores). Specifically, Study II revealed that force assistance increases the user's perception of ease of use by $19.80 \%$, usefulness by $27.03 \%$, predictability by $11.63 \%$, human-robot fluency by $22.58 \%$, goal perception by $18.75 \%$, 
robotic intelligence by $23.31 \%$, and satisfaction by $21.98 \%$.

\section{Trust}

Study II revealed that participants had a similar level of trust (not statistically different) in the robotic system among all control strategies. And this was not an exception for those with force assistance. This could be due to the fact that the robots were tele-operated, concurring with findings of trust in shared control such as in Rakita et al. (2019). As a result, and probably due to the assistance modalities not masking the users' perception of their own control, the trust levels are not significantly affected.

\subsubsection{Objective Metrics}

\section{Efficiency of Sub-tasks}

We expected force assistance to improve efficiency in all sub-tasks that required maintaining the grasp and holding the object, and in the sub-task simultaneously involving all four arms (i.e., sub-task 3.). As the grasp is more secured with force assistance, completing all sub-tasks successively should be easier for the operator.

Study II confirmed that force assistance positively improves the efficiency during the four-arm screwing task. Furthermore it has a positive impact on the sub-tasks 6. and 7.. All the other sub-tasks, however, had similar median efficiencies with no statistical differences provoked by force assistance. This could be because these sub-tasks did not challenge the user sufficiently so as to create significant delays in their execution without the force support of the robots.

\section{Number of Object Falls}

As shown in Figure 12, the strategies with force assistance, namely $U+F$ and $C+F$, provided the highest holding forces on average with relatively low variances. By generating sufficient and robust holding forces to maintain the grasp, we expected the force assistance modality to reduce significantly the number of failures. We hypothesized that the number of falls would always be lower when force assistance is used.

Results from Study II confirm our hypothesis. Indeed, force assistance substantially reduces the number of times the object will fall during the task.

\section{Completeness and Overall Success}

Regarding the completeness and success of the overall task, we suspected force assistance would always provoke a remarked increase in these metrics. Because we hypothesized increased efficiencies, and a lower number of falls, we expected a higher number of tasks completed hence all these numbers would be reflected in overall success.

Study II confirmed our hypothese,s as force assistance positively affects the task completeness and overall success of the task.

\subsection{Interaction of Force Assistance and Coordination Assistance}

Our general hypothesis was that adding force assistance to coordination assistance has a positive interaction effect. Specifically, we expected each assistance modality to compensate any downside of the other one or, in turn, to amplify its positive effect. We tested this hypothesis, based on the magnitudes and signs of the interaction coefficients of Study II. This was done specifically for the subjective metrics and not for objective metrics, because the objective ones were not normally distributed.

\subsubsection{Task Load}

The combination of force and coordination did not have a statistically significant interaction effect on any of the taskload indexes. For this reason our hypothesis is rejected. This means that for the case of TLX Physical Demand, force assistance is sufficient to enable a distinguishable lower physical demand, and does not considerably improve the interaction with coordination assistance.

\subsubsection{Fluency}

Using the constant coefficients of the interactions from study II, we found that the combination of force and coordination assistance significantly improves the perceived ease of use $(+8.37 \%)$, usefulness $(+10.81 \%)$, and predictability $(+11.63 \%)$. Therefore, for these categories, our hypotheses are correct.

The coefficients also showed that the interaction did not significantly changed the perceived human-robot fluency, goal perception, robot intelligence and satisfaction. And for these categories, our hypothesis of interaction are rejected. Indeed, it was force assistance that created a positive effect in the subjective perception of the user for these categories, and the result was not much improved when coordination assistance was added.

Finally, similarly to the main effects of force and coordination assistance, there was no statistical significance 
of the interaction of these assistance modalities on trust. For this reason our hypothesis about interaction improving trust is rejected.

\subsection{Is $C+F$ the best control strategy when compared to $U$ ?}

We hypothesized that $C+F$ would outperform $U, U+F$ and $C$ in all objective and subjective metrics. Based on the findings discussed before, there was indeed an interaction among assistance modalities in some subjective metrics. Nevertheless, we must still answer the following question: Is $C+F$ significantly better than $U+F$ and $C$ with respect to $U$ ?

Using the post-hoc analyses in Study I, we were able to answer this question both for subjective and objective metrics.

\subsubsection{Subjective Metrics}

\section{Task Load}

As discussed previously, there were statistical differences among control strategies regarding TLX Physical Demand. In this case, the responses for $U+F$ and $C+F$ (and not $C$ ) were significantly better than $U$. Nevertheless, there was no statistical difference between $U+F$ and $C+F$. For this reason, we conclude that either $U+F$ and $C+F$ could equally improve upon the $U$ control strategy. In other words, any strategy with force assistance has a statistically equal positive effect in physical demand. Therefore our hypothesis for this category is rejected.

For the rest of task-load indexes, specifically mental demand, temporal demand, performance demand, effort and frustration, there were no statistical differences with respect to $U$. Therefore our hypotheses for these categories are rejected.

\section{Fluency}

For ease of use, usefulness, robot intelligence and satisfaction, the only control strategy that was significantly better than $U$ was $C+F$. For this reason our hypothesis is correct for these categories.

This means that for predictability, perceived fluency and goal perception, using another strategy than $U$ does not improve these metrics. Nevertheless, in case the user must absolutely use a single foot to control both robots, it is crucial to have force assistance to have responses similar to a two-foot control.
Finally, as discussed before, all control strategies evoked statistically similar responses regarding trust. Hence, for this category, our hypothesis regarding $C+F$ vs $U$ is not valid either.

\subsubsection{Objective Metrics}

Efficiency and Completeness

Regarding efficiency and completeness, our hypothesis is not valid. Namely, $C+F$ does not outperform the other control strategies with respect to $U$ in these metrics. Indeed, as shown in Study II, any control strategy with force assistance has a positive effect in the efficiency of some sub-tasks (3., 6., and 7.).

\section{Number of Object Falls}

Regarding the number of object falls, $C+F$ was clearly better than the other control strategies when compared to $U$. For this reason, our hypotheses is true for this metric.

\section{Overall Success}

Finally, our hypothesis is confirmed for success as well. This means that when considering the compound metric that optimizes for higher efficiency, higher completeness, and minimum object falls, i.e., overall success, $C+F$ is the control strategy that provides significantly better results with respect to the baseline $U$.

\subsubsection{Overview}

In general, $C+F$ is the best control strategy when considering $U$ as the baseline.

\subsection{Effect of the Time-Invariant DS Framework}

Putting aside the assistance modalities, intrinsically, the tele-operation scheme used to control both robots is a standard force-reflecting bilateral tele-operation architecture (Niemeyer et al. (2016)), where the interaction forces measured by a slave robot are reflected to the master interface, whereas the slave robot tracks a reference position/velocity controlled through the master interface. Although these position-force architectures often select a PD controller for the tracking of the telemanipulator's reference commands, in this work we use the DS-based impedance controller specifically to control the robots' linear motion, according to the task (reaching, dual arm grasping and rotating the object in the sagital plane). We used the DSbased framework because it opens the door to (shared) tasks for the robot with an increasing level of complexity encoded in the time-invariant dynamical system. Indeed, DS can be exploited to generate complex smooth behaviours, for 
instance to reach a target while moving obstacles (KhansariZadeh and Billard (2012); Huber et al. (2019)) or to switch between multiple tasks (Khoramshahi and Billard (2019)). Therefore, the reactivity and adaptability of this framework makes it suitable for developing shared-control strategies in human-robot collaborative scenarios where the robot has to interact both with humans and its environment. Furthermore, as such a DS uses the feedback of the current state (e.g., position, force or other states of the system) to compute the subsequent desired velocity, it can counter any realtime disturbances, which enables immediate re-planning of the task from the disturbed state. This being said, in this work we chose to start with a simple linear DS for the uncoordinated control or baseline ( i.e., pure tele-operation ) and increased the complexity for the other assistance modalities. For example in $U+F$, we merge the motion input of the human with the autonomous-force generation in a unified DS formulation in (10) that is tracked with the same impedance control as the baseline. This is also the case for the strategies with coordination assistance, in which we couple the dynamical systems that both robotic arms are tracking. Note that, in the baseline strategy in which the attractor is the mapping of the foot's position in relation to a desired robot end effector's position, the formulation is approximately equal to a PD controller. The only difference is that the $\boldsymbol{P}$ matrix is not a longer diagonal, but it becomes state varying and task dependent. Specifically, from (7) and (3), the apparent stiffness (the gradient of the control force $\boldsymbol{F}_{\boldsymbol{R}, \boldsymbol{c}}^{\boldsymbol{i}}$ in (3) with respect to $\boldsymbol{x}^{\boldsymbol{i}}$ ) can be approximated as $\boldsymbol{P}^{i}\left(\boldsymbol{x}^{i}\right)=\boldsymbol{D}^{i}\left(\boldsymbol{x}^{i}\right) \boldsymbol{A}^{i}$, where $\boldsymbol{P}^{i}\left(\boldsymbol{x}^{i}\right) \in \mathbb{R}^{3 \times 3}$ is a virtual stiffness matrix if the simple linear DS tracking equation were to be re-formulated as a PD controller. Indeed, the state-varying damping matrix $D^{i}\left(x^{i}\right)$ of the DS-impedance law changes according to the task; specifically, the robot enables a selective compliance in the directions not relevant to the task, e.g., low gains in the directions orthogonal to the grasping direction. For this reason, considering the conditions in which the task was developed (no significant external disturbances), we believe that the results obtained and the conclusions drawn from the experimental study are agnostic to the dynamical system framework. In turn, we explain its implementation on its known robustness and flexibility for physical human robot interaction.

\subsection{Limitations and Future work}

In our work, we explored the effectiveness of contactinitiated shared control strategies in improving the subjective fluency of the human-robot interaction, as well as the task load of the participants. We also studied their effect in objective metrics of the efficiency of the sub-tasks, the number of object falls, completeness and the overall success of the task.

A positive byproduct of our experiments is the observation of coordination patterns during foot-hand manipulations (Extension 6). In our future work, we will focus on understanding better the human-motor control aspect, by quantitatively and systematically analyzing these tendencies. Thus, by basing our research on challenges and limitations of human multi-limb coordination and by aiming to achieve stable and accurate coordination, we will be able to devise assistance modalities. Furthermore, we are interested in more complex four-arm maneuvers such as those present in laparoscopic surgery. By increasing the complexity of the tasks, the assistance modalities we have proposed should also increase in complexity. For instance, force assistance appeared to be crucial in maintaining a robust grasp, the robots' alignment when grasping the object could also be a point to include in the control, to avoid unintentional rotations of the object while being grasped. Therefore, to improve the execution of the task, future explorations could also include auto-alignment of the robotic arms as an additional assistance level. Moreover, more concern should be given to the design of the experimental setup. In particular, elements such as the user's posture when performing the task, the position of the feet and the type of shoes worn could affect the comfort of the participants, when executing the task, and their perception of the task. For instance, the distance between the foot interfaces was fixed for all the participants. This could also jeopardize the execution of some sub-tasks that involved object rotations because people with long legs performed symmetric internal roll motions and had a limited range, because their knees collided (see Figure 7). For this reason, the initial position of the foot interfaces should be individually adjusted to each participant. Also, given the challenges of only having coordination assistance, the workspace and force mapping gains, between the foot platform and robot arm, might need a special adjustment for this control strategy.

We did not find any significant effects on the mental workload, possibly due to the way the robots were tasked (holding and grasping). Hence, there is a need for more manipulative tasks in the future and a need to explore deeper the role of shared control in the cognitive load for four-arm manipulation. In this respect, so far we have used the NASA-TLX to evaluate the mental workload, for which some studies have found concurrence with physiological 
measures (e.g., Borghini et al. (2012); Lecoutre et al. (2015)). Nonetheless, there can also be discrepancies (Myrtek et al. (1994); Funke et al. (2013); Di Flumeri et al. (2018)). For this reason, we would like to use state-ofthe-art non-obstructive techniques for cognitive workload assessment such as Index of Cognitive Activity (ICA) via eye-tracking (Alnæs et al. (2014); Vogels et al. (2018)).

Finally, whether in standard or shared bilateral teleoperation, it is important to guarantee a stable closed-loop behaviour, both in free motion and when interacting with unknown environments (Hokayem and Spong (2006)). To achieve stability, a sufficient condition to meet is passivity (van der Schaft and Van Der Schaft (2000)). This requires that the system never generates extra energy or that, in other words, the total energy of the system is bounded by the initially stored energy plus the one injected in the system from the interaction with the environment. In standard bilateral tele-operation, passivity has been extensively studied (Lee and Spong (2006), Franken et al. (2011)). Franken et al. (2011) introduced a general two-layer control architecture combining passivity and transparency for a bilateral tele-operation system. The idea is to explicitly separate the achievement of transparency and stability in two different layers. A top transparency layer is proposed to compute the desired control commands/torques for both the master and the slave devices. A bottom passivity layer is used to adjust the desired commands by monitoring and enforcing the energy balance of the whole system via the use of energy tanks (Secchi et al. (2006)), and by ensuring passivity and stability of the tele-operation. The intuitiveness and flexibility provided by this two-layer architecture has been recently exploited in Ferraguti et al. (2015b); Heck et al. (2018). In future work, similarly to the works above, we would like to include passivity assurance with energy tanks, to monitor the energy flow in the system and prevent the generation of extra energy coming from the control actions. As the total system is composed of two master interfaces and slave robots in our case, we could use one energy tank for each of them (four in total) and adapt their control laws to make sure that the total system stays passive.

\section{Conclusion}

In this paper, we have provided insight on the pertinence of shared-control strategies in four-arm manipulation with the particularity of using feet as user inputs. Our goal was to perform robust collaborative contact tasks with the user. For this, we incorporated shared control with contactstate information to facilitate proactively the task for the user.

Our main finding from the user study is that force assistance is, overall, perceived as better than coordination assistance in a bilaterally isomorphic task during bi-pedal telemanipulation where grasping is involved. We also find that force assistance translates into more robust manipulations and it improves the efficiency of a four-arm task where hands and feet are involved.

Finally, when combining a single-foot control with force assistance and, compared with two-foot control without force assistance, there is higher success in the task. Furthermore, the user expressed better ease of use, usefulness, impression of robot intelligence, and satisfaction during the human-robot collaboration.

\section{Acknowledgments}

The authors would like to thank all the participants to the user study.

\section{Funding}

The authors would like to thank for the support of the Hasler Foundation.

\section{References}

Abbink D and van der Helm F (2004) Force perception measurements at the foot. In: 2004 IEEE Int. Conf. Syst. Man Cybern. (IEEE Cat. No.04CH37583), volume 3. IEEE. ISBN 0-7803-8567-5, pp. 2525-2529. DOI:10.1109/ICSMC. 2004.1400709. URL http://ieeexplore.ieee.org/ document/1400709/.

Abbink DA and Mulder M (2010) Neuromuscular analysis as a guideline in designing shared control. In: Advances in haptics. IntechOpen

Abi-Farraj F, Pacchierotti C and Giordano PR (2018) User evaluation of a haptic-enabled shared-control approach for robotic telemanipulation. In: 2018 IEEE/RSJ International Conference on Intelligent Robots and Systems (IROS). IEEE, pp. 1-9.

Abi-Farraj F, Pedemonte N and Giordano PR (2016) A visual-based shared control architecture for remote telemanipulation. In: 2016 IEEE/RSJ International Conference on Intelligent Robots and Systems (IROS). IEEE, pp. 4266-4273.

Alnæs D, Sneve MH, Espeseth T, Endestad T, van de Pavert SHP and Laeng B (2014) Pupil size signals mental effort deployed 
during multiple object tracking and predicts brain activity in the dorsal attention network and the locus coeruleus. J. Vis. 14(4). DOI:10.1167/14.4.1.

Amanhoud W, Khoramshahi M and Billard A (2019) A dynamical system approach to motion and force generation in contact tasks. In: Proceedings of Robotics: Science and Systems. FreiburgimBreisgau, Germany. DOI:10.15607/RSS.2019.XV. 021.

Baldissera F, Cavallari P and Civaschi P (1982) Preferential coupling between voluntary movements of ipsilateral limbs. Neurosci. Lett. 34(1): 95-100. DOI:10.1016/0304-3940(82) 90098-2.

Boessenkool H, Abbink DA, Heemskerk CJ, van der Helm FC and Wildenbeest JG (2012) A task-specific analysis of the benefit of haptic shared control during telemanipulation. IEEE Transactions on Haptics 6(1): 2-12.

Borghini G, Vecchiato G, Toppi J, Astolfi L, Maglione A, Isabella R, Caltagirone C, Kong W, Wei D, Zhou Z et al. (2012) Assessment of mental fatigue during car driving by using high resolution eeg activity and neurophysiologic indices. In: 2012 Annual International Conference of the IEEE Engineering in Medicine and Biology Society. IEEE, pp. 6442-6445.

Brett P, Taylor R, Proops D, Coulson C, Reid A and Griffiths M (2007) A surgical robot for cochleostomy. In: 2007 29th Annual International Conference of the IEEE Engineering in Medicine and Biology Society. IEEE, pp. 1229-1232.

Di Flumeri G, Borghini G, Aricò P, Sciaraffa N, Lanzi P, Pozzi S, Vignali V, Lantieri C, Bichicchi A, Simone A et al. (2018) Eeg-based mental workload neurometric to evaluate the impact of different traffic and road conditions in real driving settings. Frontiers in human neuroscience 12: 509.

Dragan AD and Srinivasa SS (2013) A policy-blending formalism for shared control. The International Journal of Robotics Research 32(7): 790-805.

Ferraguti F, Preda N, Bonfe M and Secchi C (2015a) Bilateral teleoperation of a dual arms surgical robot with passive virtual fixtures generation. In: 2015 IEEE/RSJ International Conference on Intelligent Robots and Systems (IROS). IEEE, pp. 4223-4228.

Ferraguti F, Preda N, Manurung A, Bonfe M, Lambercy O, Gassert R, Muradore R, Fiorini P and Secchi C (2015b) An energy tank-based interactive control architecture for autonomous and teleoperated robotic surgery. IEEE Transactions on Robotics 31(5): 1073-1088.

Franken M, Stramigioli S, Misra S, Secchi C and Macchelli A (2011) Bilateral telemanipulation with time delays: A twolayer approach combining passivity and transparency. IEEE transactions on robotics 27(4): 741-756.
Fuchs K (2002) Minimally invasive surgery. Endoscopy 34(02): 154-159.

Funke G, Knott B, Mancuso VF, Strang A, Estepp J, Menke L, Brown R, Dukes A and Miller B (2013) Evaluation of subjective and eeg-based measures of mental workload. In: International Conference on Human-Computer Interaction. Springer, pp. 412-416.

Geitner C, Birrell S, Krehl C and Jennings P (2018) Haptic Foot Pedal: Influence of Shoe Type, Age, and Gender on Subjective Pulse Perception. Hum. Factors J. Hum. Factors Ergon. Soc. 60(4): 496-509. DOI:10.1177/0018720818771906.

URL http://www.ncbi.nlm.nih.gov/pubmed/ 29768043 http: // journals.sagepub.com/doi/ $10.1177 / 0018720818771906$.

Goil A, Derry M and Argall BD (2013) Using machine learning to blend human and robot controls for assisted wheelchair navigation. In: 2013 IEEE 13th International Conference on Rehabilitation Robotics (ICORR). IEEE, pp. 1-6.

Gopinath D, Jain S and Argall BD (2016) Human-in-the-loop optimization of shared autonomy in assistive robotics. IEEE robotics and automation letters 2(1): 247-254.

Griffin WB, Provancher WR and Cutkosky MR (2005) Feedback strategies for telemanipulation with shared control of object handling forces. Presence: Teleoperators \& Virtual Environments 14(6): 720-731.

Guthart GS and Salisbury JK (2000) The intuitive/sup tm/telesurgery system: overview and application. In: Proceedings 2000 ICRA. Millennium Conference. IEEE International Conference on Robotics and Automation. Symposia Proceedings (Cat. No. O0CH37065), volume 1. IEEE, pp. 618-621.

Hansson A and Servin M (2010) Semi-autonomous shared control of large-scale manipulator arms. Control Engineering Practice 18(9): 1069-1076.

Hart SG and Staveland LE (1988) Development of nasa-tlx (task load index): Results of empirical and theoretical research. In: Advances in psychology, volume 52. Elsevier, pp. 139-183.

Heck D, Saccon A, Beerens R and Nijmeijer H (2018) Direct forcereflecting two-layer approach for passive bilateral teleoperation with time delays. IEEE Transactions on Robotics 34(1): 194206.

Hernandez Sanchez J, Amanhoud W, Haget A, Bleuler H, Billard A and Bouri M (2019) Four-Arm Manipulation via Feet Interfaces. In: Assoc. Adv. Artif. Intell. Fall Symp.Series. (AAAI - FSS). Arlington, Virginia. URL http://arxiv.org/ abs/1909.04993.

Hirche S, Stanczyk B and Buss M (2003) Transparent exploration of remote environments by internet telepresence. In: Proceedings 
of Int. Workshop on High-Fidelity Telepresence and Teleaction jointly with the conference HUMANOIDS.

Hoffman G (2019) Evaluating Fluency in Human-Robot Collaboration. IEEE Trans. Human-Machine Syst. 49(3): 209-218. DOI: 10.1109/THMS.2019.2904558.

Hokayem PF and Spong MW (2006) Bilateral teleoperation: An historical survey. Automatica 42(12): 2035-2057.

Huber L, Billard A and Slotine JJ (2019) Avoidance of convex and concave obstacles with convergence ensured through contraction. IEEE Robotics and Automation Letters 4(2): 1462-1469.

Hunter I and Kearney R (1982) Dynamics of human ankle stiffness: Variation with mean ankle torque. J. Biomech. 15(10): 747-752. DOI:10.1016/0021-9290(82)90089-6. URL https: / / www.sciencedirect.com/science/ article/pii/0021929082900896.

Ichinose A, Gomikawa Y and Suzuki S (2013) Driving assistance through pedal reaction force control with consideration of JND. In: 2013 IEEE RO-MAN. IEEE. ISBN 978-1-4799-0509-6, pp. 484-489. DOI:10.1109/ROMAN.2013.6628551. URL http: //ieeexplore.ieee.org/document/6628551/.

Kearney R, Weiss P and Morier R (1990) System identification of human ankle dynamics: intersubject variability and intrasubject reliability. Clin. Biomech. 5(4): 205217. DOI:10.1016/0268-0033(90)90004-P. URL https://www.sciencedirect.com/science/ article/pii/026800339090004P.

Kelso JA and Jeka JJ (1992) Symmetry breaking dynamics of human multilimb coordination. J. Exp. Psychol. Hum. Percept. Perform. 18(3): 645-668. DOI:10.1037//0096-1523.18.3.645.

Khansari-Zadeh SM and Billard A (2012) A dynamical system approach to realtime obstacle avoidance. Autonomous Robots 32(4): 433-454.

Khoramshahi M and Billard A (2019) A dynamical system approach to task-adaptation in physical human-robot interaction. Autonomous Robots 43(4): 927-946.

Khoramshahi M, Laurens A, Triquet T and Billard A (2018) From human physical interaction to online motion adaptation using parameterized dynamical systems. In: 2018 IEEE/RSJ International Conference on Intelligent Robots and Systems (IROS). IEEE, pp. 1361-1366.

Khurshid RP, Fitter NT, Fedalei EA and Kuchenbecker KJ (2016) Effects of grip-force, contact, and acceleration feedback on a teleoperated pick-and-place task. IEEE transactions on haptics 10(1): 40-53.

Kim WS, Tendick F, Ellis SR and Stark LW (1987) A Comparison of Position and Rate Control for Telemanipulations with Consideration of Manipulator System Dynamics. IEEE
J. Robot. Autom. 3(5): 426-436. DOI:10.1109/JRA.1987. 1087117.

Kronander K and Billard A (2015) Passive interaction control with dynamical systems. IEEE Robotics and Automation Letters 1(1): 106-113.

Laghi M, Maimeri M, Marchand M, Leparoux C, Catalano M, Ajoudani A and Bicchi A (2018) Shared-autonomy control for intuitive bimanual tele-manipulation. In: 2018 IEEE-RAS 18th International Conference on Humanoid Robots (Humanoids). IEEE, pp. 1-9.

Lecoutre L, Lini S, Bey C, Lebour Q and Favier PA (2015) Evaluating eeg measures as a workload assessment in an operational video game setup. In: PhyCS. pp. 112-117.

Lee D and Spong MW (2006) Passive bilateral teleoperation with constant time delay. IEEE transactions on robotics 22(2): 269281.

Mirrazavi S, Khoramshahi M and Billard A (2016) A dynamical system approach for catching softly a flying object: Theory and experiment. IEEE Transactions on Robotics 32.

Muelling K, Venkatraman A, Valois JS, Downey JE, Weiss J, Javdani S, Hebert M, Schwartz AB, Collinger JL and Bagnell JA (2017) Autonomy infused teleoperation with application to brain computer interface controlled manipulation. Autonomous Robots 41(6): 1401-1422.

Myrtek M, Deutschmann-Janicke E, Strohmaier H, Zimmermann W, Lawerenz S, Brügner G and Müller W (1994) Physical, mental, emotional, and subjective workload components in train drivers. Ergonomics 37(7): 1195-1203.

Niemeyer G, Preusche C, Stramigioli S and Lee D (2016) Telerobotics. In: Springer handbook of robotics. Springer, pp. 1085-1108.

Parietti F, Chan KC, Hunter B and Asada HH (2015) Design and control of supernumerary robotic limbs for balance augmentation. In: 2015 IEEE International Conference on Robotics and Automation (ICRA). IEEE, pp. 5010-5017.

Passenberg C, Peer A and Buss M (2010) A survey of environment, operator-, and task-adapted controllers for teleoperation systems. Mechatronics 20(7): 787-801.

Rakita D, Mutlu B, Gleicher M and Hiatt LM (2019) Shared control-based bimanual robot manipulation. Science Robotics 4(30). DOI:10.1126/scirobotics.aaw0955. URL https://robotics.sciencemag.org/content/ 4/30/eaaw0955.

Roelofsen EG, Bosga J, Rosenbaum DA, Nijhuis-van der Sanden MW, Hullegie W, van Cingel R and Meulenbroek RG (2016) Haptic feedback helps bipedal coordination. Exp. Brain Res. 234(10): 2869-2881. DOI:10.1007/s00221-016-4689-2. URL http://www.ncbi.nlm.nih.gov/pubmed/ 
27263085http: //www.pubmedcentral.nih.gov/ articlerender.fcgi?artid=PMC5025491.

Rosenberg LB (1993) Virtual fixtures: Perceptual tools for telerobotic manipulation. In: Proceedings of IEEE virtual reality annual international symposium. IEEE, pp. 76-82.

Salehian SSM and Billard A (2018) A dynamical system based approach for controlling robotic manipulators during noncontact/contact transitions. IEEE Robotics and Automation Letters .

Sanes JN and Truccolo W (2003) Motor "Binding:" do functional assemblies in primary motor cortex have a role? DOI:10.1016/ S0896-6273(03)00203-4.

Secchi C, Stramigioli S and Fantuzzi C (2006) Position drift compensation in port-hamiltonian based telemanipulation. In: 2006 IEEE/RSJ International Conference on Intelligent Robots and Systems. IEEE, pp. 4211-4216.

Segura Meraz N, Sobajima M, Aoyama T and Hasegawa Y (2018) Modification of body schema by use of extra robotic thumb. ROBOMECH J. 5(1): 3. DOI:10.1186/s40648-018-0100-3. URL https://robomechjournal.springeropen. com/articles/10.1186/s40648-018-0100-3.

Selvaggio M, Abi-Farraj F, Pacchierotti C, Giordano PR and Siciliano B (2018) Haptic-based shared-control methods for a dual-arm system. IEEE Robotics and Automation Letters 3(4): 4249-4256.

Selvaggio M, Chen F, Gao B, Notomista G, Trapani F and Caldwell D (2016) Vision based virtual fixture generation for teleoperated robotic manipulation. In: 2016 International Conference on Advanced Robotics and Mechatronics (ICARM). IEEE, pp. 190-195.

Serrien DJ and Swinnen SP (1997) Coordination Constraints Induced by Effector Combination under Isofrequency and Multifrequency Conditions. J. Exp. Psychol. Hum. Percept. Perform. 23(5): 1493-1510. DOI:10.1037/0096-1523.23.5. 1493.

Sheridan TB (1993) Space teleoperation through time delay: Review and prognosis. IEEE Transactions on robotics and Automation 9(5): 592-606.

Shirkhodaie A (2002) Supervised control of cooperative multiagent robotic vehicles. In: Proceedings of the ThirtyFourth Southeastern Symposium on System Theory (Cat. No. 02EX540). IEEE, pp. 386-390.

Siegler S, Chen J and Schneck CD (1988) The Three-Dimensional Kinematics and Flexibility Characteristics of the Human Ankle and Subtalar Joints-Part I: Kinematics. J. Biomech. Eng. 110(4): 364. DOI:10.1115/1.3108455. URL http: //biomechanical. asmedigitalcollection . asme.org/article.aspx?articleid=1398022.
Smisek J, van Paassen M and Schiele A (2015) Haptic guidance in bilateral teleoperation: Effects of guidance inaccuracy. In: 2015 IEEE World Haptics Conference (WHC). IEEE, pp. 500-505.

Storms JG and Tilbury DM (2014) Blending of human and obstacle avoidance control for a high speed mobile robot. In: 2014 American Control Conference. IEEE, pp. 3488-3493.

Swinnen SP, Jardin K, Meulenbroek R, Dounskaia N and Van Den Brandt MH (1997) Egocentric and allocentric constraints in the expression of patterns of interlimb coordination. J. Cogn. Neurosci. 9(3): 348-377. DOI:10.1162/jocn. 1997.9.3.348. URL http://www.ncbi.nlm.nih.gov/ pubmed/23965012.

Swinnen SP and Wenderoth N (2004) Two hands, one brain: Cognitive neuroscience of bimanual skill. DOI:10.1016/j.tics. 2003.10.017.

van der Schaft AJ and Van Der Schaft A (2000) L2-gain and passivity techniques in nonlinear control, volume 2 . Springer. van Oosterhout J, Wildenbeest JG, Boessenkool H, Heemskerk CJ, de Baar MR, van der Helm FC and Abbink DA (2015) Haptic shared control in tele-manipulation: Effects of inaccuracies in guidance on task execution. IEEE transactions on haptics 8(2): 164-175.

Vogels J, Demberg V and Kray J (2018) The Index of Cognitive Activity as a Measure of Cognitive Processing Load in Dual Task Settings. Front. Psychol. 9(NOV): 2276. DOI:10.3389/ fpsyg.2018.02276. URL https://www. frontiersin. org/article/10.3389/fpsyg.2018.02276/full.

Wang X, Yang C, Ma H and Cheng L (2015) Shared control for teleoperation enhanced by autonomous obstacle avoidance of robot manipulator. In: 2015 IEEE/RSJ International Conference on Intelligent Robots and Systems (IROS). IEEE, pp. 4575-4580. 


\section{A Index to Multimedia Extensions}

\begin{tabular}{|c|c|l|}
\hline $\begin{array}{c}\text { Extension } \\
\text { Number }\end{array}$ & $\begin{array}{c}\text { Media } \\
\text { type }\end{array}$ & \multicolumn{1}{|c|}{ Title } \\
\hline 1 & Video & $\begin{array}{l}\text { Demonstration of the 4A Task } \\
\text { and the Control Strategy U }\end{array}$ \\
\hline 2 & Video & $\begin{array}{l}\text { Demonstration of the } \\
\text { Control Strategy U+F }\end{array}$ \\
\hline 3 & Video & $\begin{array}{l}\text { Demonstration of the } \\
\text { Control Strategy C+F }\end{array}$ \\
\hline 4 & Video & $\begin{array}{l}\text { Demonstration of the } \\
\text { Control Strategy C }\end{array}$ \\
\hline 5 & Video & $\begin{array}{l}\text { Demonstration of the Degrees } \\
\text { of Freedom Mappings } \\
\text { for Uncoordinated vs } \\
\text { Coordinated Control }\end{array}$ \\
\hline 6 & Video & $\begin{array}{l}\text { Remarks on Human } \\
\text { Motor Control }\end{array}$ \\
\hline
\end{tabular}

Table 1. Index of the multimedia extensions

\section{B Technical Details}

- For all participants, each control strategy runs at a frequency of $200 \mathrm{~Hz}$ (i.e., $d t=0.005 \mathrm{~s}$ ).

- For both robotic arms, the control gains were set to

$-d_{1}^{i}=150$.

- $\boldsymbol{K}_{\boldsymbol{o}}^{\boldsymbol{i}}=\operatorname{diag}([15,15,15])$.

- $\boldsymbol{D}_{\boldsymbol{o}}^{\boldsymbol{i}}=\operatorname{diag}([2,2,2])$.

- For both of the foot interfaces, we set the control gains to:

- $\boldsymbol{K}_{\boldsymbol{M}}^{\boldsymbol{i}}=\operatorname{diag}([0,0,0,3500,1000])$.

- $\boldsymbol{D}_{M}^{i}=\operatorname{diag}([3,5,20,60,30])$.

- To compute the axis-angle orientation error $\tilde{\zeta}^{i}$ between $z_{o}^{i}$ and $z_{o, d}^{i}$, we proceed in two steps. We first use the Rodrigues' rotation formula to compute the rotation matrix $\tilde{R}$ aligning $z_{o}^{i}$ with $z_{o, d}^{i}$ :

$$
\left\{\begin{array}{l}
c=z_{o}^{i^{T}} z_{o, d}^{i} \\
k=z_{o}^{i} \times z_{o, d}^{i} \\
s=\|\boldsymbol{k}\| \\
\boldsymbol{K}=[\boldsymbol{k}]_{\times} \\
\tilde{\boldsymbol{R}}=\boldsymbol{I}_{\mathbf{3} \times \mathbf{3}}+s \boldsymbol{K}+(1-c) \boldsymbol{K}^{\mathbf{2}}
\end{array}\right.
$$

We then convert $\tilde{\boldsymbol{R}}$ into $\tilde{\zeta}^{i}$.

- The desired angular velocity $\boldsymbol{\omega}_{\boldsymbol{d}}^{\boldsymbol{i}}$ is decomposed into two terms:

$$
\omega_{d}^{i}=\omega_{d, \zeta}+\omega_{d, h}
$$

- $\omega_{d, \zeta}$ helps aligning $z_{o}^{i}$ with $z_{o, d}^{i}$. To this end, the axis-angle error is converted into a unit rotation quaternion $\tilde{Q}^{i}$ in order to obtain a desired quaternion $Q_{d}^{i}=\tilde{Q}^{i} \star Q^{i}$ where $\star$ denotes the quaternion product, and $Q^{i}$ is the measured robot quaternion. Using the relationship between angular velocity and quaternion time derivative, we obtain $\omega_{d, \zeta}$ through:

$$
Q_{\omega}=k_{\omega} Q^{i^{-1}} \star\left(Q_{d}^{i}-Q^{i}\right)
$$

where $k_{\omega}$ is a positive gain set to 5 , and $\boldsymbol{Q}_{\boldsymbol{\omega}}=$ $\left[\begin{array}{ll}0 & \boldsymbol{\omega}_{\boldsymbol{d}, \boldsymbol{\zeta}}^{\boldsymbol{E}^{T}}\end{array}\right]^{T} . \boldsymbol{\omega}_{\boldsymbol{d}, \boldsymbol{\zeta}}^{\boldsymbol{E}}$ is $\boldsymbol{\omega}_{\boldsymbol{d}, \boldsymbol{\zeta}}$ expressed in the endeffector frame. To express it back in the world frame, we simply use the end-effector orientation matrix.

- $\omega_{d, h}$ is the component commanded by the human to rotate the robots' end-effector/object:

$$
\boldsymbol{\omega}_{\boldsymbol{d}, \boldsymbol{h}}= \begin{cases}\omega_{h}^{i} \boldsymbol{z}_{\boldsymbol{o}}^{\boldsymbol{i}} & \text { strategy } U / U+F \\ \omega_{h} \boldsymbol{n}^{\boldsymbol{j}} & \text { strategy } C / C+F\end{cases}
$$

where $j$ is the id of the foot platform used to control both robots in the coordinated strategies.

- For control strategy $U$, we set

- $\boldsymbol{A}^{\boldsymbol{i}}=\operatorname{diag}([2,2,2])$.

- $\Upsilon_{\boldsymbol{p}}^{U}=\left[\begin{array}{ccccc}0 & \frac{2 R_{x}}{r_{y}} & 0 & 0 & 0 \\ -\frac{2 R_{y}}{r_{x}} & 0 & 0 & 0 & 0 \\ 0 & 0 & \frac{2 R_{z}}{r_{\theta}} & 0 & 0 \\ 0 & 0 & 0 & \frac{2 R_{\omega}}{r_{\phi}} & 0\end{array}\right]$, where $R_{x}$ $=R_{y}=0.4 \mathrm{~m}, R_{z}=0.3 \mathrm{~m}, R_{\omega}=1.5 \mathrm{rad}$ are desired ranges of motion on the robots' side, and $r_{x}=0.195$ $m, r_{y}=0.18 m, r_{\theta}=48^{\circ}$, and $r_{\phi}=40^{\circ}$ are ranges of motion on the foot interface.

- $x_{o}^{i}=[-0.5,0.45,0.2]^{T}$.

- $\boldsymbol{z}_{\boldsymbol{o}, \boldsymbol{d}}^{\boldsymbol{L}}=[0,-1,0]^{T}$ and $\boldsymbol{z}_{\boldsymbol{o}, \boldsymbol{d}}^{\boldsymbol{R}}=[0,1,0]^{T}$.

$-\Upsilon_{\boldsymbol{F}}^{U}=\alpha\left[\begin{array}{ccc}0 & -s_{x} & 0 \\ s_{y} & 0 & 0 \\ 0 & 0 & s_{\theta} \\ 0 & 0 & 0 \\ 0 & 0 & 0\end{array}\right]$, where $\alpha=1, s_{x}=1, s_{y}=$ 1 , and $s_{\theta}=0.2$ are scaling factors. 
- For control strategy $U+F$, we set

- $F_{T}^{U, i}=30 \mathrm{~N}$.

- $\varepsilon_{F}=5 N$ and $n=10$.

- $\Upsilon_{p}^{U}$ is equal to above except $\alpha=0.7$.

- All the other variables as in control strategy $U$.

- For control strategy $C+F$, we set

- $\boldsymbol{A}_{\boldsymbol{C}}=\operatorname{diag}([3,3,3])$ and $\boldsymbol{A}_{\boldsymbol{D}}=\operatorname{diag}([2,2,2])$.

- $P=\left[\begin{array}{lll}0 & 0 & 0 \\ 0 & 1 & 0 \\ 0 & 0 & 0\end{array}\right]$.

- $\boldsymbol{\Upsilon}_{\boldsymbol{F}}^{U}$ is similar to above except $\alpha=0.7$.

- $F_{T}^{C, L}=F_{T}\left(1+\Upsilon_{a, b}\left(d_{1}^{R} \dot{\boldsymbol{x}}_{\boldsymbol{d}}^{C^{\boldsymbol{T}}} \boldsymbol{n}^{\boldsymbol{R}}+30\right)\right)$ and $F_{T}^{C, R}=F_{T}\left(1+\Upsilon_{a, b}\left(d_{1}^{L} \dot{\boldsymbol{x}}_{\boldsymbol{d}}^{C^{\boldsymbol{T}}} \boldsymbol{n}^{\boldsymbol{L}}+F_{T}\right)\right)$ with $a$ $=10 \mathrm{~N}, \mathrm{~b}=15 \mathrm{~N}, F_{T}=30 \mathrm{~N}$, and where $\Upsilon_{a, b}(x)$ is a function providing a smooth transition from 0 to 1 as $x$ transits from $a$ to $b$ :

$$
\Upsilon_{a, b}(x)= \begin{cases}0 & x<a \\ \frac{1}{2}\left(1-\cos \left(\pi \frac{x-a}{b-a}\right)\right) & a \leq x \leq b \\ 1 & x>b\end{cases}
$$

The target force for each robot is modulated to account for the balance between the motion force in the grasping direction (i.e., $d_{1}^{i} \dot{x}_{d}^{C^{T}} n^{i}$ ) and the nominal contact force $F_{T}$ of the other robot. When moving along the grasping direction, one robot would have its motion and target-contact forces opposite to each other. As a result, the applied contact force would reduce and the grasp might be broken. To limit this effect, we modulate the target force of the other robot to apply more force (up to twice the nominal one). This keeps the object within the robots' grasp, without reducing the robots' velocity.

- $\dot{x}_{h, l i m}=0.3 \mathrm{~m} / \mathrm{s}$ and $\omega_{h, l i m}=1.5 \mathrm{rad} / \mathrm{s}$.

- The right foot is used for all participants to control both robots in coordination: $j=R$.

$-\Upsilon_{\boldsymbol{p}}^{C}=\left[\begin{array}{ccccc}0 & \frac{2 R_{x}}{r_{y}} & 0 & 0 & 0 \\ -\frac{2 R_{y}}{r_{x}} & 0 & 0 & 0 & 0 \\ 0 & 0 & \frac{2 R_{z}}{r_{\theta}} & 0 & 0 \\ 0 & 0 & 0 & \frac{2 R_{\omega}}{r_{\phi}} & 0 \\ 0 & 0 & 0 & 0 & \frac{2 R_{\dot{x}}}{r_{\psi}}\end{array}\right]$, where $R_{\dot{x}}=0.3 \mathrm{~m} / \mathrm{s}, r_{\psi}=40^{\circ}$, while the other variables are equal to before.

$-x_{o}^{C}=x_{o}^{R}-\frac{x_{0}^{D}}{2}$.
- $\Upsilon_{\boldsymbol{F}}^{C}=\alpha\left[\begin{array}{ccc}0 & 0 & 0 \\ 0 & 0 & 0 \\ 0 & 0 & 0 \\ 0 & 0 & 0 \\ 0 & s_{\psi} & 0\end{array}\right]$, where $\alpha=0.7$ and $s_{\psi}=1$.

- $\varepsilon_{D}=\left\|\boldsymbol{x}_{\mathbf{0}}^{\boldsymbol{D}}\right\|+0.2 \mathrm{~m}$ and $\varepsilon_{x}=0.07 \mathrm{~m}$.

$-\boldsymbol{\Omega}_{\boldsymbol{F}}^{\boldsymbol{C}}=k\left[\begin{array}{ccc}0 & -s_{x} & 0 \\ s_{y} & 0 & 0 \\ 0 & 0 & s_{\theta} \\ 0 & 0 & 0 \\ 0 & 0 & 0\end{array}\right]$, where $k=200$ while $s_{x}=$ $1, s_{y}=1$, and $s_{\theta}=0.2$.

- For control strategy $C$, we set:

- $\mathbf{\Upsilon}_{\boldsymbol{F}}^{\boldsymbol{C}}$ is equal to above except $\alpha=1$.

- All the other variables as in control strategy $C+F$. 